\title{
ASYMPTOTIC ERGODICITY OF THE EIGENVALUES OF RANDOM OPERATORS IN THE LOCALIZED PHASE
}

\author{
FRÉDÉRIC KLOPP
}

\begin{abstract}
We prove that, for a general class of random operators, the family of the unfolded eigenvalues in the localization region is asymptotically ergodic in the sense of N. Minami (see [25]). N. Minami conjectured this to be the case for discrete Anderson model in the localized regime. We also provide a local analogue of this result. From the asymptotics ergodicity, one can recover the statistics of the level spacings as well as a number of other spectral statistics. Our proofs rely on the analysis developed in [12].

RÉSumÉ. On démontre que, pour une classe générale d'opérateurs aléatoires, les familles valeurs propres "dépliées" sont asymptotiquement ergodiques au sens de N. Minami (voir [25]). N. Minami à conjecturé que ceci est vrai pour le modèle d'Anderson discret dans le régime localisé. On démontre également un résultat analogue pour les valeurs propres "locales". L'ergodicité asymptotique des valeurs propres permet alors d'en déduire les statistiques des espacements de niveaux ainsi que nombre d'autres statistiques spectrales. Nos preuves reposent sur l'analyse faite dans [12].
\end{abstract}

\section{INTRODUCTION}

On $\ell^{2}\left(\mathbb{Z}^{d}\right)$, consider the random Anderson model

$$
H_{\omega}=-\Delta+\lambda V_{\omega}
$$

where

- $-\Delta$ is the free discrete Laplace operator

$$
(-\Delta u)_{n}=\sum_{|m-n|=1} u_{m} \quad \text { for } u=\left(u_{n}\right)_{n \in \mathbb{Z}^{d}} \in \ell^{2}\left(\mathbb{Z}^{d}\right) ;
$$

- $V_{\omega}$ is the random potential

$$
\left(V_{\omega} u\right)_{n}=\omega_{n} u_{n} \quad \text { for } u=\left(u_{n}\right)_{n \in \mathbb{Z}^{d}} \in \ell^{2}\left(\mathbb{Z}^{d}\right) .
$$

We assume that the random variables $\left(\omega_{n}\right)_{n \in \mathbb{Z}^{d}}$ are independent identically distributed and that their common distribution admits a compactly supported bounded density, say $g$.

- The coupling constant $\lambda$ is chosen positive.

It is then well known (see e.g. [18]) that

- let $\Sigma:=[-2 d, 2 d]+\operatorname{supp} g$ and $S_{-}$and $S_{+}$be the infimum and supremum of $\Sigma$; for almost every $\omega=\left(\omega_{n}\right)_{n \in \mathbb{Z}^{d}}$, the spectrum of $H_{\omega}$ is equal to $\Sigma$;

- there exists a bounded density of states, say $E \mapsto \nu(E)$, such that, for any continuous function $\varphi: \mathbb{R} \rightarrow \mathbb{R}$, one has

$$
\int_{\mathbb{R}} \varphi(E) \nu(E) d E=\mathbb{E}\left(\left\langle\delta_{0}, \varphi\left(H_{\omega}\right) \delta_{0}\right\rangle\right)
$$

The author is partially supported by the grant ANR-08-BLAN-0261-01. 
Here, and in the sequel, $\mathbb{E}(\cdot)$ denotes the expectation with respect to the random parameters, and $\mathbb{P}(\cdot)$ the probability measure they induce.

Let $N$ be the integrated density of states of $H_{\omega}$ i.e. $N$ is the distribution function of the measure $\nu(E) d E$. The function $\nu$ is only defined $E$-almost everywhere. In the sequel, when we speak of $\nu(E)$ for some $E$, we mean that the non decreasing function $N$ is differentiable at $E$ and that $\nu(E)$ is its derivative at $E$.

For $L \in \mathbb{N}$, let $\Lambda=\Lambda_{L}=[-L, L]^{d}$ be a large box and $|\Lambda|:=\# \Lambda=(2 L+1)^{d}$ be its cardinality. Let $H_{\omega}(\Lambda)$ be the operator $H_{\omega}$ restricted to $\Lambda$ with periodic boundary conditions. The notation $|\Lambda| \rightarrow+\infty$ is a shorthand for considering $\Lambda=\Lambda_{L}$ in the limit $L \rightarrow+\infty$. Let us denote the eigenvalues of $H_{\omega}(\Lambda)$ ordered increasingly and repeated according to multiplicity by $E_{1}(\omega, \Lambda) \leq E_{2}(\omega, \Lambda) \leq \cdots \leq E_{|\Lambda|}(\omega, \Lambda)$.

For $t \in[0,1]$, consider the following point process

$$
\Xi(\omega, t, \Lambda)=\sum_{n=1}^{|\Lambda|} \delta_{|\Lambda|\left[N\left(E_{n}(\omega, \Lambda)\right)-t\right]} .
$$

We prove

Theorem 0.1. For sufficiently large coupling constant $\lambda$, $\omega$-almost surely, when $|\Lambda| \rightarrow$ $+\infty$, the probability law of the point process $\Xi(\omega, \cdot, \Lambda)$ under the uniform distribution $\mathbf{1}_{[0,1]}(t) d t$ converges to the law of the Poisson point process on the real line with intensity 1 .

This proves in particular a conjecture by N. Minami (see [22, 25]); a weaker version of Theorem 0.1, namely, $L^{2}$-convergence in $\omega$ when $d=1$, is proved in [25].

Theorem 0.1, in particular, implies the convergence of the level spacings statistics already obtained for this model under more restrictive assumptions in [12] (see also Theorem 1.3 in the present paper for more details). Indeed, in Theorem 0.1, we do not make any regularity assumption on the distribution of the random variables except for their having a common bounded compactly supported density.

Actually, Theorem 0.1 is a prototype of the general result we state and prove below. Essentially, we prove that the claim in Theorem 0.1 holds in the localization region for any random Hamiltonian satisfying a Wegner and a Minami estimate (see assumptions (W) and (M) in section 1). To do so, we use the analysis made in [12]; in particular, our analysis relies on one of the approximation theorems proved in [12], namely, Theorem 1.16.

\section{The Results}

Consider $H_{\omega}=H_{0}+V_{\omega}$, a $\mathbb{Z}^{d}$-ergodic random Schrödinger operator on $\mathcal{H}=L^{2}\left(\mathbb{R}^{d}\right)$ or $\ell^{2}\left(\mathbb{Z}^{d}\right)$ (see e.g. [26, 28]). Typically, the background potential $H_{0}$ is the Laplacian $-\Delta$, possibly perturbed by a periodic potential. Magnetic fields can be considered as well; in particular, the Landau Hamiltonian is also admissible as a background Hamiltonian. For the sake of simplicity, we assume that $V_{\omega}$ is almost surely bounded; hence, almost surely, $H_{\omega}$ have the same domain $H^{2}\left(\mathbb{R}^{d}\right)$ or $\ell^{2}\left(\mathbb{Z}^{d}\right)$.

1.1. The setting and the assumptions. For $\Lambda$, a cube in either $\mathbb{R}^{d}$ or $\mathbb{Z}^{d}$, we let $H_{\omega}(\Lambda)$ be the self-adjoint operator $H_{\omega}$ restricted to $\Lambda$ with periodic boundary conditions. As in [12], our analysis stays valid for Dirichlet boundary conditions.

Furthermore, we shall denote by $\mathbf{1}_{J}(H)$ the spectral projector of the operator $H$ on the energy interval $J . \mathbb{E}(\cdot)$ denotes the expectation with respect to $\omega$. 
Our first assumption will be an independence assumption for local Hamiltonians that are far away from each other, that is,

(IAD): There exists $R_{0}>0$ such that for any two cubes $\Lambda$ and $\Lambda^{\prime}$ such that $\operatorname{dist}\left(\Lambda, \Lambda^{\prime}\right)>R_{0}$, the random Hamiltonians $H_{\omega}(\Lambda)$ and $H_{\omega}\left(\Lambda^{\prime}\right)$ are stochastically independent.

Remark 1.1. This assumption may be relaxed to assume that the correlation between the random Hamiltonians $H_{\omega}(\Lambda)$ and $H_{\omega}\left(\Lambda^{\prime}\right)$ decays sufficiently fast as $\operatorname{dist}\left(\Lambda, \Lambda^{\prime}\right) \rightarrow+\infty$. We refer to [12] for more details.

Let $\Sigma$ be the almost sure spectrum of $H_{\omega}$. Pick $I$ a relatively compact open subset of $\Sigma$. Assume the following holds:

(W): a Wegner estimate holds in $I$, i.e. there exists $C>0$ such that, for $J \subset I$, and $\Lambda$, a cube in $\mathbb{R}^{d}$ or $\mathbb{Z}^{d}$, one has

$$
\mathbb{E}\left[\operatorname{tr}\left(\mathbf{1}_{J}\left(H_{\omega}(\Lambda)\right)\right)\right] \leq C|J||\Lambda| .
$$

(M): a Minami estimate holds in $I$, i.e. there exists $C>0$ and $\rho>0$ such that, for $J \subset I$, and $\Lambda$, a cube in $\mathbb{R}^{d}$ or $\mathbb{Z}^{d}$, one has

$$
\mathbb{E}\left[\operatorname{tr}\left(\mathbf{1}_{J}\left(H_{\omega}(\Lambda)\right)\right) \cdot\left[\operatorname{tr}\left(\mathbf{1}_{J}\left(H_{\omega}(\Lambda)\right)\right)-1\right]\right] \leq C(|J||\Lambda|)^{1+\rho} .
$$

Remark 1.2. The Wegner estimate (W) has been proved for many random Schrödinger models e.g. for both discrete and continuous Anderson models under rather general conditions on the single site potential and on the randomness (see e.g. [16, 18, 19, 29]) but also for other models (see e.g. [14, 21]). The right hand side in (1.1) can be lower bounded by the probability to have at least one eigenvalue in $J$ (for $J$ small).

Weaker forms of assumption (W) i.e. when the right hand side is replaced with $C|J|^{\alpha}|\Lambda|^{\beta}$ for some $\alpha \in(0,1]$ and $\beta \geq 1$, are known to hold also for some non monotonous models (see e.g. $[20,17,13])$. This is sufficient for our proofs to work if one additionnally knows that the integrated density of states is absolutely continuous.

On the Minami estimate (M), much less is known: in any dimension, it holds for the discrete Anderson model with $I=\Sigma$ (see [23, 15, 3, 6]). For the continuous Anderson model in any dimension, in [7], it is shown to hold at the bottom of the spectrum under more restrictive conditions on the single site potential than needed to prove the Wegner estimate $(\mathrm{W})$. These proofs yield an optimal exponent $\rho=1$. The right hand side in (1.2) can be lower bounded by the probability to have at least two eigenvalues in $J$. So, (M) can be interpreted as a measure of the independence of close by eigenvalues.

The integrated density of states is defined as

$$
N(E):=\lim _{|\Lambda| \rightarrow+\infty} \frac{\#\left\{\text { e.v. of } H_{\omega}(\Lambda) \text { less than } \mathrm{E}\right\}}{|\Lambda|}
$$

By $(\mathrm{W}), N(E)$ is the distribution function of a measure that is absolutely continuous with respect to to the Lebesgue measure on $\mathbb{R}$. Let $\nu$ be the density of state of $H_{\omega}$ i.e. the distributional derivative of $N$. In the sequel, for a set $I,|N(I)|$ denotes the Lebesgue measure of $N(I)$ i.e. $|N(I)|=\int_{I} \nu(E) d E$.

Let us now describe what we call the localized regime in the introduction. For $L \geq 1, \Lambda_{L}$ denotes the cube $[-L / 2, L / 2]^{d}$ in either $\mathbb{R}^{d}$ or $\mathbb{Z}^{d}$. In the sequel, we write $\Lambda$ for $\Lambda_{L}$ i.e. $\Lambda=\Lambda_{L}$ and when we write $|\Lambda| \rightarrow+\infty$, we mean $L \rightarrow+\infty$. 
Let $\mathcal{H}_{\Lambda}$ be $\ell^{2}\left(\Lambda \cap \mathbb{Z}^{d}\right)$ in the discrete case and $L^{2}(\Lambda)$ in the continuous one. For a vector $\varphi \in \mathcal{H}$, we define

$$
\|\varphi\|_{x}=\left\{\begin{array}{cl}
\left\|\mathbf{1}_{\Lambda(x)} \varphi\right\|_{2} \text { where } \Lambda(x)=\{y ;|y-x| \leq 1 / 2\} & \text { if } \mathcal{H}=L^{2}\left(\mathbb{R}^{d}\right), \\
|\varphi(x)| & \text { if } \mathcal{H}=\ell^{2}\left(\mathbb{Z}^{d}\right) .
\end{array}\right.
$$

Let $I$ be a compact interval. We assume that $I$ lies in the region of complete localization (see e.g. $[10,11])$ for which we use the following finite volume version:

(Loc): for all $\xi \in(0,1)$, one has

$$
\sup _{L>0} \sup _{\substack{\sup f \subset I \\|f| \leq 1}} \mathbb{E}\left(\sum_{\gamma \in \mathbb{Z}^{d}} e^{|\gamma| \xi}\left\|\mathbf{1}_{\Lambda(0)} f\left(H_{\omega}\left(\Lambda_{L}\right)\right) \mathbf{1}_{\Lambda(\gamma)}\right\|_{2}\right)<+\infty .
$$

Remark 1.3. Such a region of localization has been shown to exist and described for many random models (see e.g. [11, 2, 1, 28, 20, 17, 13, 14, 21]); a fairly recent review can be found in [18]; other informational texts include [26, 10].

Once a Wegner estimate is known (though it is not an absolute requirement see e.g. [5, 9, 8]), the typical regions where localization holds are vicinities of the edges of the spectrum. One may have localization over larger regions (or the whole) of the spectrum if the disorder is large like in Theorem 0.1.

This assumption (Loc) may be relaxed; we refer to Remark 1.3 of [12] for more details.

For $L \in \mathbb{N}$, recall that $\Lambda=\Lambda_{L}$ and that $H_{\omega}(\Lambda)$ is the operator $H_{\omega}$ restricted to $\Lambda$ with periodic boundary conditions. The notation $|\Lambda| \rightarrow+\infty$ is a shorthand for considering $\Lambda=\Lambda_{L}$ in the limit $L \rightarrow+\infty$.

Finally, let $E_{1}(\omega, \Lambda) \leq E_{2}(\omega, \Lambda) \leq \cdots \leq E_{N}(\omega, \Lambda) \leq \cdots$ denote the eigenvalues of $H_{\omega}(\Lambda)$ ordered increasingly and repeated according to multiplicity.

We state our results in two cases. In the first case described in section 1.2 , we consider a macroscopic energy interval i.e. the energy interval in which we study the eigenvalues is a fixed compact interval where all the above assumptions hold. In the second case described in section 1.3, the energy interval shrinks to a point but not too fast so as to contain enough eigenvalues that is asymptotically infinitely many eigenvalues.

We also consider another point of view on the random Hamiltonian. Namely, under assumption (Loc), in $I$, one typically proves that the spectrum is made only of eigenvalues and that to these eigenvalues, one associates exponentially decaying eigenfunctions (exponential or Anderson localization) (see e.g. [26, 10, 11, 18]). One can then enumerate these eigenvalues in an energy interval by considering only those with localization center (i.e. with most of their mass) in some cube $\Lambda$ and study the thus obtained process. This is done in section 1.4 .

1.2. Macroscopic energy intervals. For $J=[a, b]$ a compact interval such that $N(b)-$ $N(a)=|N(J)|>0$ and a fixed configuration $\omega$, consider the point process

$$
\Xi_{J}(\omega, t, \Lambda)=\sum_{E_{n}(\omega, \Lambda) \in J} \delta_{|N(J)||\Lambda|\left[N_{J}\left(E_{n}(\omega, \Lambda)\right)-t\right]}
$$

under the uniform distribution in $[0,1]$ in $t$; here we have set

$$
N_{J}(\cdot):=\frac{N(\cdot)-N(a)}{N(b)-N(a)}=\frac{N(\cdot)-N(a)}{|N(J)|} .
$$

Our main result is 
Theorem 1.1. Assume (IAD), (W), (M) and (Loc) hold. Assume that $J \subset I$, the localization region, is such that $|N(J)|>0$.

Then, $\omega$-almost surely, the probability law of the point process $\Xi_{J}(\omega, \cdot, \Lambda)$ under the uniform distribution $\mathbf{1}_{[0,1]}(t) d t$ converges to the law of the Poisson point process on the real line with intensity 1.

First, let us note that Theorem 0.1 is an immediate consequence of Theorem 1.1 as it is well known that, for the discrete Anderson model at large disorder, the whole spectrum is localized in the sense of (Loc) (see e.g. [18]).

A number of spectral statistics for the sequence of unfolded eigenvalues are immediate consequences of Theorem 1.1 and the results of [24]. For example, by Proposition 4.4 of [24], it implies the convergence of the empirical distribution of unfolded level spacings to $e^{-x}$ (see [24, 25, 12]). We refer to [24] for more results on the statistics of asymptotically ergodic sequences.

As in [12], one can also study the statistics of the levels themselves i.e. before unfolding. Using classical results on transformations of point processes (see [4, 27]) and the fact that $N$ is Lipschitz continuous and increasing, one obtains

Theorem 1.2. Assume (IAD), (W), (M) and (Loc) hold. Assume that $J=[a, b] \subset I$ is a compact interval in the localization region satisfying $|N(J)|>0$.

Define

- the probability density $\nu_{J}:=\frac{1}{|N(J)|} \nu(t) \mathbf{1}_{J}(t)$ where $\nu=\frac{d N}{d E}$ is the density of states of $H_{\omega}$;

- the point process $\tilde{\Xi}_{J}(\omega, t, \Lambda)=\sum_{E_{n}(\omega, \Lambda) \in J} \delta_{\nu(t)|\Lambda|\left[E_{n}(\omega, \Lambda)-t\right]}$.

Then, $\omega$-almost surely, the probability law of the point process $\tilde{\Xi}_{J}(\omega, \cdot, \Lambda)$ under the distribution $\nu_{J}(t) d t$ converges to the law of the Poisson point process on the real line with intensity 1 .

We note that, in Theorem 1.2, we don't make any regularity assumption on $N$ except for the Wegner estimate. This enables us to remove the regularity condition imposed on the density of states $\nu$ in the proof of the almost sure convergence of the level spacings statistics given in [12]. Thus, we prove

Theorem 1.3. Assume (IAD), (W), (M) and (Loc) hold. Pick $J \subset I$ a compact interval in the localization region such that $|N(J)|>0$. Let $N(J, \omega, \Lambda)$ be the random number of eigenvalues of $H_{\omega}(\Lambda)$ is $J$. Define the eigenvalue or level spacings as

$$
\forall 1 \leq j \leq N(J, \omega, \Lambda), \quad \delta_{J} E_{j}(\omega, \Lambda)=\frac{|N(J)|}{|J|}|\Lambda|\left(E_{j+1}(\omega, \Lambda)-E_{j}(\omega, \Lambda)\right) \geq 0
$$

and the empirical distribution of these spacings to be the random numbers, for $x \geq 0$

$$
D L S(x ; J, \omega, \Lambda)=\frac{\#\left\{j ; E_{j}(\omega, \Lambda) \in J, \delta_{J} E_{j}(\omega, \Lambda) \geq x\right\}}{N(J, \omega, \Lambda)} .
$$

Then, $\omega$-almost surely, as $|\Lambda| \rightarrow+\infty, D L S^{\prime}(x ; J, \omega, \Lambda)$ converges uniformly to the distribution $x \mapsto g_{\nu, J}(x)$ where $g_{\nu, J}(x)=\int_{J} e^{-\nu_{J}(\lambda)|J| x} \nu_{J}(\lambda) d \lambda$. 
1.3. Microscopic energy intervals. One can also prove a version of Theorem 1.1 that is local in energy. In this case, one needs that the weight the density of states puts on the energy interval under consideration not be too small with respect to the length of the energy interval (see the first condition in (1.8)). One proves

Theorem 1.4. Assume (IAD), (W), (M) and (Loc) hold. Pick $E_{0} \in I$.

Fix $\left(I_{\Lambda}\right)_{\Lambda}$ a decreasing sequence of intervals such that $\sup _{I_{\Lambda}}|x| \underset{|\Lambda| \rightarrow+\infty}{\rightarrow} 0$. Assume that, for some $\delta>0$ and $\tilde{\rho} \in(0, \rho /(1+(1+\rho) d)$ ) (recall that $\rho$ is defined in $(M))$, one has

$$
N\left(E_{0}+I_{\Lambda}\right) \cdot\left|I_{\Lambda}\right|^{-1-\tilde{\rho}} \geq 1, \quad|\Lambda|^{1-\delta} \cdot N\left(E_{0}+I_{\Lambda}\right) \underset{|\Lambda| \rightarrow+\infty}{\rightarrow}+\infty
$$

and

$$
\text { if } \ell^{\prime}=o(L) \text { then } \frac{N\left(E_{0}+I_{\Lambda_{L+\ell^{\prime}}}\right)}{N\left(E_{0}+I_{\Lambda_{L}}\right)} \underset{L \rightarrow+\infty}{\rightarrow} 1 \text {. }
$$

Then, $\omega$-almost surely, the probability law of the point process $\Xi_{E_{0}+I_{\Lambda}}(\omega, \cdot, \Lambda)$ under the uniform distribution $\mathbf{1}_{[0,1]}(t) d t$ converges to the law of the Poisson point process on the real line with intensity 1.

Note that the first condition in (1.8) requires that the derivative of $N$ does not vanish too fast at $E_{0}$. As a consequence of Theorem 1.4, using the results of [24], one shows that one has convergence of the unfolded local level spacings distribution at any point of the almost sure spectrum if one looks at "large" enough neighborhoods of the point; here, "large" does not mean that the neighborhood needs to be large: it merely needs not to shrink too fast to 0 (see (1.8)).

1.4. Results for the random Hamiltonian on the whole space. In our previous results, we considered the eigenvalues of the random Hamiltonian restricted to a box. As in [12], one can also consider the operator $H_{\omega}$ on the whole space. Therefore, we recall

Proposition 1.1 ([12]). Assume (IAD), (W) and (Loc). Fix $q>2 d$. Then, there exists $\gamma>0$ such that, $\omega$-almost surely, there exists $C_{\omega}>1, \mathbb{E}\left(C_{\omega}\right)<\infty$, such that

(1) with probability 1 , if $E \in I \cap \sigma\left(H_{\omega}\right)$ and $\varphi$ is a normalized eigenfunction associated to $E$ then, for some $x(E, \omega) \in \mathbb{R}^{d}$ or $\mathbb{Z}^{d}$, a maximum of $x \mapsto\|\varphi\|_{x}$, for some $C_{\omega}>0$, one has, for $x \in \mathbb{R}^{d}$,

$$
\|\varphi\|_{x} \leq C_{\omega}\left(1+|x(E, \omega)|^{2}\right)^{q / 2} e^{-\gamma|x-x(E, \omega)|^{\xi}}
$$

where $\|\cdot\|_{x}$ is defined in (1.4).

Moreover, one has $\mathbb{E}\left(C_{\omega}\right)<+\infty$.

$x(E, \omega)$ is a center of localization for $E$ or $\varphi$.

(2) Pick $J \subset I$ such that $|N(J)|>0$. Let $N^{f}(J, \Lambda, \omega)$ denotes the number of eigenvalues of $H_{\omega}$ having a center of localization in $\Lambda$. Then, there exists $\beta>0$ such that, for $\Lambda$ sufficiently large, one has

$$
\left|\frac{N^{f}(J, \Lambda, \omega)}{|N(J)||\Lambda|}-1\right| \leq \frac{1}{\log ^{\beta}|\Lambda|} .
$$

In view of Proposition 1.1, $\omega$-almost surely, there are only finitely many eigenvalues of $H_{\omega}$ in $J$ having a localization center in $\Lambda_{L}$. Thus, we can enumerate these eigenvalues as $E_{1}^{f}(\omega, \Lambda) \leq E_{2}^{f}(\omega, \Lambda) \leq \cdots \leq E_{N}^{f}(\omega, \Lambda)$ where we repeat them according to multiplicity. For $t \in[0,1]$, define the point process $\Xi_{J}^{f}(\omega, t, \Lambda)$ by (1.6) and (1.7) for those eigenvalues. As a corollary of Theorem 1.1, we obtain 
Theorem 1.5. Assume (IAD), (W), (M) and (Loc) hold. Assume that $J \subset I$, the localization region, that $|N(J)|>0$.

Then, $\omega$-almost surely, the probability law of the point process $\Xi_{J}^{f}(\omega, \cdot, \Lambda)$ under the uniform distribution $\mathbf{1}_{[0,1]}(t) d t$ converges to the law of the Poisson point process on the real line with intensity 1.

Theorem 1.5 also admits an corresponding analogue that is local in energy i.e. a counterpart of Theorem 1.4.

1.5. Outline of the paper. Let us briefly outline the remaining parts of the paper. In section 2, we recall some results from [12] that we build our analysis upon. The strategy of the proof will be roughly to study the eigenvalues of the random operator where the integrated density of states, $N(\cdot)$, takes value close to $t$. Most of those eigenvalues, as in shown in [12], can be approximated by i.i.d. random variables the distribution law of which is roughly uniform on $[0,1]$ when properly renormalized. We then show that this approximation is accurate enough to obtain the almost sure convergence announced in Theorem 1.1.

Theorem 1.4 is proved in the same way and we only make a few remarks on this proof in section 3.6. Theorem 1.5 is deduced from Theorem 1.1 approximating the eigenvalues of $H_{\omega}$ by those of $H_{\omega}(\Lambda)$ for sufficiently large $\Lambda$; this is done in section 3.7.

Section 4 is devoted to the proof of Theorems 1.2 and 1.3 . It relies on point process techniques, in particular, on transformations of point processes (see e.g. [4, 27]).

\section{The SPECTRUM OF A RANDOM OPERATOR IN THE LOCALIZED REGIME}

Let us now recall some results taken from [12] that will use in our proofs.

2.1. Distribution of the unfolded eigenvalues. We now describe the distribution of the unfolded eigenvalues for the operator $H_{\omega}$ in a small cube. Pick $1 \ll \ell^{\prime} \ll \ell$. Consider a cube $\Lambda=\Lambda_{\ell}$ centered at 0 of side length $\ell$. Pick an interval $I_{\Lambda}=\left[a_{\Lambda}, b_{\Lambda}\right] \subset I$ (i.e. $I_{\Lambda}$ is contained in the localization region) for $\ell$ sufficiently large.

Consider the following random variables:

- $X=X\left(\Lambda, I_{\Lambda}\right)=X\left(\Lambda, I_{\Lambda}, \ell^{\prime}\right)$ is the Bernoulli random variable

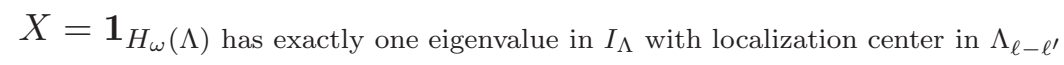

- $\tilde{E}=\tilde{E}\left(\Lambda, I_{\Lambda}\right)$ is this eigenvalue conditioned on $X=1$.

Let $\tilde{\Xi}$ be the distribution function of $\tilde{E}$. We know

Lemma 2.1 ([12]). Assume (W), (M) and (Loc) hold.

For $\kappa \in(0,1)$, one has

$$
|\mathbb{P}(X=1)-| N\left(I_{\Lambda}\right)|| \Lambda|| \lesssim\left(|\Lambda|\left|I_{\Lambda}\right|\right)^{1+\rho}+\left|N\left(I_{\Lambda}\right)\right||\Lambda| \ell^{\prime} \ell^{-1}+|\Lambda| e^{-\left(\ell^{\prime}\right)^{\kappa}}
$$

where $N(E)$ denotes the integrated density of states of $H_{\omega}$.

One has

$$
|(\tilde{\Xi}(x)-\tilde{\Xi}(y)) P(X=1)| \lesssim|x-y|\left|I_{\Lambda}\right||\Lambda| .
$$

Moreover, setting $N(x, y, \Lambda):=\left[N\left(a_{\Lambda}+x\left|I_{\Lambda}\right|\right)-N\left(a_{\Lambda}+y\left|I_{\Lambda}\right|\right)\right]|\Lambda|$, one has

$$
\begin{aligned}
|(\tilde{\Xi}(x)-\tilde{\Xi}(y)) P(X=1)-N(x, y, \Lambda)| & \\
& \lesssim\left(|\Lambda|\left|I_{\Lambda}\right|\right)^{1+\rho}+|N(x, y, \Lambda)| \ell^{\prime} \ell^{-1}+|\Lambda| e^{-\left(\ell^{\prime}\right)^{\kappa}} .
\end{aligned}
$$


Estimates (2.1) and (2.2) are of interest mainly if their right hand side, which is to be understood as an error term, is smaller than the main term. In (2.1), the main restriction comes from the requirement that $N\left(I_{\Lambda}\right)|\Lambda| \gg\left(|\Lambda|\left|I_{\Lambda}\right|\right)^{1+\rho}$ which is essentially a requirement that $N\left(I_{\Lambda}\right)$ should not be too small with respect to $\left|I_{\Lambda}\right|$. Lemma 2.1 will be used in conjunction with Theorems 2.1. The cube $\Lambda$ in Lemma 2.1 will be the cube $\Lambda_{\ell}$ in Theorem 2.1. Therefore, the requirements induced by the other two terms are less restrictive. The second term is an error term if $\ell^{\prime} \ll \ell$ which is guaranteed by assumption; this induces no new requirement. The third term in the right hand side of (2.1) being small compared to $\left|N\left(I_{\Lambda}\right)\right||\Lambda|$ requires that $\left|N\left(I_{\Lambda}\right)\right||\Lambda| \gg \ell^{d} e^{-\left(\ell^{\prime}\right)^{\kappa}}$. This links the size of the cube $\Lambda=\Lambda_{\ell}$ where we apply Lemma 2.1 to the size of $\left|N\left(I_{\Lambda}\right)\right|$. The right choice for $\ell$ (that will become clear from Theorem 2.1 stated below) is $\ell \asymp\left|N\left(I_{\Lambda}\right)\right|^{-\nu}$. In our application, we will pick $\ell^{\prime} \asymp(\log \ell)^{1 / \xi}$ for some $\xi \in(0,1)$ (coming from the localization estimate (Loc)); so taking $\kappa>\xi$ ensures that the third term in the right hand side of (2.1) is small compared to $\left|N\left(I_{\Lambda}\right)\right||\Lambda|$. For further details, we refer to the comments following the statement of Theorem 2.1 and section 3.2 for details.

In (2.2), the main restriction comes from the requirement that $N(x, y, \Lambda)|\Lambda| \gg\left(|\Lambda|\left|I_{\Lambda}\right|\right)^{1+\rho}$. This is essentially a requirement on the size of $|x-y|$. It should not be too small. On the other hand, we expect the spacing between the eigenvalues of $H_{\omega}\left(\Lambda_{L}\right)$ to be of size $\left|\Lambda_{L}\right|^{-1}$ (we keep the notations of Theorem 2.1 and recall that the cube $\Lambda$ in Lemma 2.1 will be the cube $\Lambda_{\ell}$ in Theorem 2.1, hence, a cube much smaller that $\Lambda_{L}$ ). So to distinguish between the eigenvalues, one needs to be able to know $\tilde{\Xi}$ up to resolution $|x-y|\left|I_{\Lambda}\right| \sim\left|\Lambda_{L}\right|^{-1}$. This will force us to use Lemma 2.1 on intervals $I_{\Lambda}$ such that $\left|N\left(I_{\Lambda}\right)\right| \asymp|\Lambda|^{-\alpha}$ for some $\alpha \in(0,1)$ close to 1 (see the discussion following Theorem 2.1 and section 3.2). Moreover, the approximation of $\tilde{\Xi}(x)-\tilde{\Xi}(y)$ by $N(x, y, \Lambda) / P(X=1)$ will be good if $|x-y| \gg\left(\left|\Lambda_{L}\right|\left|I_{\Lambda}\right|\right)^{-1} \asymp\left|\Lambda_{L}\right|^{-\beta}$ for some $\beta>0$.

2.2. I.I.D approximations to the eigenvalues. The second ingredient of our proof is a description of most of the eigenvalues of $H_{\omega}(\Lambda)$ in some small interval, say, $I_{\Lambda}$ in terms of i.i.d. random variables. These random variables are the eigenvalues of the restrictions of $H_{\omega}(\Lambda)$ to much smaller disjoint cubes, the distribution of which we computed in Lemma 2.1. This description of the eigenvalues of $H_{\omega}(\Lambda)$ holds with a probability close to 1 .

2.2.1. Localization estimates and localization centers. We first recall a result of [12] defining and describing localization centers, namely,

Lemma $2.2([12])$. Under assumptions $(W)$ and (Loc), for any $p>0$ and $\xi \in(0,1)$, there exists $q>0$ such that, for $L \geq 1$ large enough, with probability larger than $1-L^{-p}$, if

(1) $\varphi_{n, \omega}$ is a normalized eigenvector of $H_{\omega}\left(\Lambda_{L}\right)$ associated to $E_{n, \omega} \in I$,

(2) $x_{n}(\omega) \in \Lambda_{L}$ is a maximum of $x \mapsto\left\|\varphi_{n, \omega}\right\|_{x}$ in $\Lambda_{L}$,

then, for $x \in \Lambda_{L}$, one has

$$
\left\|\varphi_{n, \omega}\right\|_{x} \leq L^{q} e^{-\left|x-x_{n}(\omega)\right|^{\xi}}
$$

where $\|\cdot\|_{x}$ is defined in (1.4).

Define $C(\varphi)=\left\{x \in \Lambda ;\|\varphi\|_{x}=\max _{\gamma \in \Lambda}\|\varphi\|_{\gamma}\right\}$ to be the set of localization centers for $\varphi$. Then, the diameter of $C\left(\varphi_{j}(\omega, \Lambda)\right)$ is less than $C_{q}(\log |\Lambda|)^{1 / \xi}$.

We define localization centers in a unique way by ordering the set $C(\varphi)$ lexicographically and take the supremum. 
2.2.2. An approximation theorem for eigenvalues. Pick $\xi \in(0,1), R>1$ large and $\rho^{\prime} \in$ $(0, \rho)$ where $\rho$ is defined in $(\mathrm{M})$. For a cube $\Lambda$, consider an interval $I_{\Lambda}=\left[a_{\Lambda}, b_{\Lambda}\right] \subset I$. Set $\ell_{\Lambda}^{\prime}=(R \log |\Lambda|)^{\frac{1}{\xi}}$. We say that the sequence $\left(I_{\Lambda}\right)_{\Lambda}$ is $\left(\xi, R, \rho^{\prime}\right)$-admissible if, for any $\Lambda$, one has

$$
|\Lambda|\left|N\left(I_{\Lambda}\right)\right| \geq 1, \quad\left|N\left(I_{\Lambda}\right)\right|\left|I_{\Lambda}\right|^{-\left(1+\rho^{\prime}\right)} \geq 1, \quad \mid N\left(I_{\Lambda}\right)^{\frac{1}{1+\rho^{\prime}}}\left(\ell_{\Lambda}^{\prime}\right)^{d} \leq 1 .
$$

One has

Theorem 2.1 ([12]). Assume (IAD), (W), (M) and (Loc) hold. Let $\Lambda=\Lambda_{L}$ be the cube of center 0 and side length $L$.

Pick $\rho^{\prime} \in[0, \rho /(1+(\rho+1) d))$ where $\rho$ is defined in $(M)$. Pick a sequence of intervals

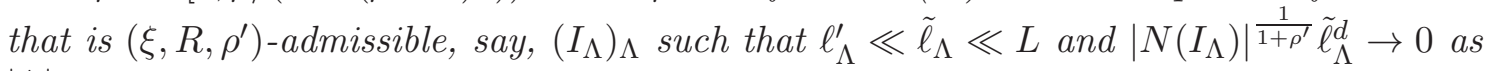
$|\Lambda| \rightarrow \infty$.

For any $p>0$, for $L$ sufficiently large (depending only on $\left(\xi, R, \rho^{\prime}, p\right)$ but not on the admissible sequence of intervals), there exists

- a decomposition of $\Lambda_{L}$ into disjoint cubes of the form $\Lambda_{\ell_{\Lambda}}\left(\gamma_{j}\right):=\gamma_{j}+\left[0, \ell_{\Lambda}\right]^{d}$, where $\ell_{\Lambda}=\tilde{\ell}_{\Lambda}\left(1+\mathcal{O}\left(\tilde{\ell}_{\Lambda} /\left|\Lambda_{L}\right|\right)\right)=\tilde{\ell}_{\Lambda}(1+o(1))$ such that

$-\cup_{j} \Lambda_{\ell_{\Lambda}}\left(\gamma_{j}\right) \subset \Lambda_{L}$,

$-\operatorname{dist}\left(\Lambda_{\ell_{\Lambda}}\left(\gamma_{j}\right), \Lambda_{\ell_{\Lambda}}\left(\gamma_{k}\right)\right) \geq \ell_{\Lambda}^{\prime}$ if $j \neq k$,

$-\operatorname{dist}\left(\Lambda_{\ell_{\Lambda}}\left(\gamma_{j}\right), \partial \Lambda_{L}\right) \geq \ell_{\Lambda}^{\prime}$,

- $\left|\Lambda_{L} \backslash \cup_{j} \Lambda_{\ell_{\Lambda}}\left(\gamma_{j}\right)\right| \lesssim\left|\Lambda_{L}\right| \ell_{\Lambda}^{\prime} / \ell_{\Lambda}$,

- a set of configurations $\mathcal{Z}_{\Lambda}$ such that

$-\mathcal{Z}_{\Lambda}$ is large, namely,

$$
\mathbb{P}\left(\mathcal{Z}_{\Lambda}\right) \geq 1-\frac{1}{2}|\Lambda|^{-p}-\exp \left(-c\left|I_{\Lambda}\right|^{1+\rho}|\Lambda| \ell_{\Lambda}^{d \rho}\right)-\exp \left(-c|\Lambda|\left|I_{\Lambda}\right| \ell_{\Lambda}^{\prime} \ell_{\Lambda}^{-1}\right)
$$

so that

- for $\omega \in \mathcal{Z}_{\Lambda}$, there exists at least $\frac{|\Lambda|}{\ell_{\Lambda}^{d}}\left(1+O\left(\left|N\left(I_{\Lambda}\right)\right|^{1 /\left(1+\rho^{\prime}\right)} \ell_{\Lambda}^{d}\right)\right)$ disjoint boxes $\Lambda_{\ell_{\Lambda}}\left(\gamma_{j}\right)$ satisfying the properties:

(1) the Hamiltonian $H_{\omega}\left(\Lambda_{\ell_{\Lambda}}\left(\gamma_{j}\right)\right)$ has at most one eigenvalue in $I_{\Lambda}$, say, $E_{n}\left(\omega, \Lambda_{\ell_{\Lambda}}\left(\gamma_{j}\right)\right)$;

(2) $\Lambda_{\ell_{\Lambda}}\left(\gamma_{j}\right)$ contains at most one center of localization, say $x_{k_{j}}(\omega, L)$, of an eigenvalue of $H_{\omega}(\Lambda)$ in $I_{\Lambda}$, say $E_{k_{j}}(\omega, \Lambda)$;

(3) $\Lambda_{\ell_{\Lambda}}\left(\gamma_{j}\right)$ contains a center $x_{k_{j}}(\omega, \Lambda)$ if and only if $\sigma\left(H_{\omega}\left(\Lambda_{\ell_{\Lambda}}\left(\gamma_{j}\right)\right)\right) \cap I_{\Lambda} \neq \emptyset$; in which case, one has

$$
\left|E_{k_{j}}(\omega, \Lambda)-E_{n}\left(\omega, \Lambda_{\ell_{\Lambda}}\left(\gamma_{j}\right)\right)\right| \leq|\Lambda|^{-R} \text { and } \operatorname{dist}\left(x_{k_{j}}(\omega, L), \Lambda_{L} \backslash \Lambda_{\ell_{\Lambda}}\left(\gamma_{j}\right)\right) \geq \ell_{\Lambda}^{\prime}
$$

where we recall that $\ell_{\Lambda}^{\prime}=(R \log |\Lambda|)^{\frac{1}{\xi}}$;

- the number of eigenvalues of $H_{\omega}(\Lambda)$ that are not described above is bounded by

$$
C\left|N\left(I_{\Lambda}\right)\right||\Lambda|\left(\left|N\left(I_{\Lambda}\right)\right|^{\frac{\rho-\rho^{\prime}}{1+\rho^{\prime}}} \ell_{\Lambda}^{d(1+\rho)}+\left|N\left(I_{\Lambda}\right)\right|^{-\frac{\rho^{\prime}}{1+\rho^{\prime}}}\left(\ell_{\Lambda}^{\prime}\right)^{d+1} \ell_{\Lambda}^{-1}\right) ;
$$

this number is $o\left(\left|N\left(I_{\Lambda}\right)\right||\Lambda|\right)$ provided

$$
\left|N\left(I_{\Lambda}\right)\right|^{-\frac{\rho^{\prime}}{1+\rho^{\prime}}}\left(\ell_{\Lambda}^{\prime}\right)^{d+1} \ll \ell_{\Lambda} \ll\left|N\left(I_{\Lambda}\right)\right|^{-\frac{\rho-\rho^{\prime}}{d(1+\rho)\left(1+\rho^{\prime}\right)}} .
$$

We note that the assumptions on $\left(I_{\Lambda}\right)_{\Lambda}$ in Theorem 2.1 imply that $\left|I_{\Lambda}\right| \rightarrow 0$ and $\left|N\left(I_{\Lambda}\right)\right|$ must go to 0 faster than logarithmically in $|\Lambda|$ (see the right hand side of (2.7)).

Let us now briefly explain how the lengthscale $\ell=\ell_{\Lambda}$ will be chosen in our analysis (see section 3.2). We will use Theorem 2.1 on intervals $I_{\Lambda}$ such that $\left|N\left(I_{\Lambda}\right)\right|=\asymp|\Lambda|^{-\alpha}$ (for some 
$\alpha \in(0,1)$ close to 1$)$ and set $\ell_{\Lambda} \asymp\left|N\left(I_{\Lambda}\right)\right|^{-\nu}$ for some $\nu \in(0,1)$. Thus, $\log \ell_{\Lambda}^{\prime} \ll \log \ell_{\Lambda}$ and checking of the validity of $(2.7)$ reduces to checking that $\frac{\rho^{\prime}}{1+\rho^{\prime}}<\frac{\rho-\rho^{\prime}}{d(1+\rho)\left(1+\rho^{\prime}\right)}$ which follow from the assumption $\rho^{\prime} \in[0, \rho /(1+(\rho+1) d))$. The exponent $\nu$ is then chosen so that

$$
\frac{\rho^{\prime}}{1+\rho^{\prime}}<\nu<\frac{\rho-\rho^{\prime}}{d(1+\rho)\left(1+\rho^{\prime}\right)}
$$

Note that the right hand side inequality in (2.7) implies that $\left|N\left(I_{\Lambda}\right)\right|^{\frac{1}{1+\rho^{\prime}}} \tilde{\ell}_{\Lambda}^{d} \rightarrow 0$ as $(\rho-$ $\left.\rho^{\prime}\right) /(1+\rho)<1$. With these choices, the bound (2.6) then becomes $\left|N\left(I_{\Lambda}\right)\right||\Lambda|^{1-\beta}$ for some $\beta>0$.

To conclude this section, we note that, when the length scales are chosen as just indicated, one easily checks that the estimate (2.4) becomes

$$
\mathbb{P}\left(\mathcal{Z}_{\Lambda}\right) \geq 1-|\Lambda|^{-p}
$$

2.3. A large deviation principle for the eigenvalue counting function. Define the random numbers

$$
N\left(I_{\Lambda}, \Lambda, \omega\right):=\#\left\{j ; E_{j}(\omega, \Lambda) \in I_{\Lambda}\right\} .
$$

Write $I_{\Lambda}=\left[a_{\Lambda}, b_{\Lambda}\right]$ and recall that $\left|N\left(I_{\Lambda}\right)\right|=N\left(b_{\Lambda}\right)-N\left(a_{\Lambda}\right)$ where $N$ is the integrated density of states. Using Theorem 2.1 and standard large deviation estimates for i.i.d. random variables, one shows that $N\left(I_{\Lambda}, \Lambda, \omega\right)$ satisfies a large deviation principle, namely,

Theorem 2.2. Assume (IAD), (W), (M) and (Loc) hold. For any $\rho^{\prime} \in(0, \rho /(1+(1+\rho) d))$ ( $\rho$ is defined in Assumption $(M)) \delta \in(0,1)$ and $\nu \in(0,1-\delta)$, there exists $\delta^{\prime}>0$ such that, if $\left(I_{\Lambda}\right)_{\Lambda}$ is a sequence of compact intervals in the localization region I satisfying

(1) $\left|N\left(I_{\Lambda}\right)\right||\Lambda|^{\delta} \rightarrow 0$ as $|\Lambda| \rightarrow+\infty$

(2) $\left|N\left(I_{\Lambda}\right)\right||\Lambda|^{1-\nu} \rightarrow+\infty$ as $|\Lambda| \rightarrow+\infty$

(3) $\left|N\left(I_{\Lambda}\right)\right|\left|I_{\Lambda}\right|^{-1-\rho^{\prime}} \rightarrow+\infty$ as $|\Lambda| \rightarrow+\infty$,

then, for any $p>0$, for $|\Lambda|$ sufficiently large (depending on $\rho^{\prime}$ and $\nu$ but not on the specific sequence $\left.\left(I_{\Lambda}\right)_{\Lambda}\right)$, one has

$$
\mathbb{P}\left(\left|N\left(I_{\Lambda}, \Lambda, \omega\right)-\right| N\left(I_{\Lambda}\right)|| \Lambda|| \geq\left|N\left(I_{\Lambda}\right)\right||\Lambda|^{1-\delta^{\prime}}\right) \leq|\Lambda|^{-p} .
$$

This result is essentially Theorem 1.8 in [12]; the only change is a change of scale for $\left|N\left(I_{\Lambda}\right)\right|$ in terms of $|\Lambda|$ (see point (1)). Up to this minor difference, the proofs of the two results are the same.

Assume that, for $J$, an interval in the region of localization $I$, one has the lower bound $|N(x)-N(y)| \gtrsim|x-y|^{1+\rho^{\prime}}$ for $(x, y) \in I^{2}$ and some $\rho^{\prime} \in(0, \rho /(1+(1+\rho) d))$. Then, as $K \mapsto|N(K)|$ is a measure, thus, additive, for $K \subset J$ the region of localization, one may split $K$ into intervals $\left(K_{k}\right)_{k}$ such that $\left|N\left(K_{k}\right)\right| \asymp|\Lambda|^{-\delta}$, and sum the estimates given by Theorem 2.2 on each $K_{k}$ to obtain that

$$
\mathbb{P}\left(|N(K, \Lambda, \omega)-| N(K)|| \Lambda|| \geq|N(K)||\Lambda|^{1-\delta^{\prime}}\right) \lesssim|\Lambda|^{-p}
$$

Though we will not need it, this gives an interesting large deviation estimate for intervals of macroscopic size. 


\section{The proofs of Theorems 1.1, 1.5 And 1.4}

We first prove Theorem 1.1. Theorem 1.5 is then a immediate consequence of Theorem 1.1 and the fact that most of the eigenvalues of $H_{\omega}(\Lambda)$ and those of $H_{\omega}$ having center of localization in $\Lambda$ differ at most by $L^{-p}$ for any $p$ and $L$ sufficiently large (see section 3.7). Theorem 1.4 is proved in the same way as Theorem 1.1 in section 3.7; we skip most of the details of this proof.

We shall use the following standard notations: $a \lesssim b$ means there exists $c<\infty$ so that $a \leq c b ;\langle x\rangle=\left(1+|x|^{2}\right)^{\frac{1}{2}}$. We write $a \asymp b$ when $a \lesssim b$ and $b \lesssim a$.

From now on, to simplify notations, we write $N$ instead of $N_{J}$ so that the density of states increases from 0 to 1 on $J$. We also write $\Xi$ instead of $\Xi_{J}$

For $\varphi: \mathbb{R} \rightarrow \mathbb{R}$ continuous and compactly supported, set

$$
\mathcal{L}_{\omega, \Lambda}(\varphi):=\mathcal{L}_{\omega, J, \Lambda}:=\int_{0}^{1} e^{-\langle\Xi(\omega, t, \Lambda), \varphi\rangle} d t
$$

and

$$
\langle\Xi(\omega, t, \Lambda), \varphi\rangle:=\sum_{E_{n}(\omega, \Lambda) \in J} \varphi\left(|\Lambda|\left[N\left(E_{n}(\omega, \Lambda)\right)-t\right]\right)
$$

To prove Theorems 1.1 and 1.4, it suffices (see [25]) to prove

Theorem 3.1. For $\varphi: \mathbb{R} \rightarrow \mathbb{R}^{+}$continuously differentiable and compactly supported, $\omega$-almost surely,

$$
\mathcal{L}_{\omega, \Lambda}(\varphi) \underset{|\Lambda| \rightarrow+\infty}{\rightarrow} \exp \left(-\int_{-\infty}^{+\infty}\left(1-e^{-\varphi(x)}\right) d x\right) .
$$

Then, a standard dense subclass argument shows that the limit (3.3) holds for compactly supported, continuous, non negative functions. This completes the proof of Theorem 1.1.

3.1. The proof of Theorem 3.1. The integrated density of states $N$ is non decreasing. By assumption (W), it is Lipschitz continuous. One can partition $[0,1]=\cup_{n \in \mathcal{N}} I_{n}$ where $\mathcal{N}$ is at most countable and $\left(I_{n}\right)_{n \in \mathcal{N}}$ are intervals such that either

- $I_{n}$ is open and $N$ is strictly increasing on the open interval $N^{-1}\left(I_{n}\right)$; we then say that $n \in \mathcal{N}^{+}$;

- $I_{n}$ reduces to a single point and $N$ is constant on the closed interval $N^{-1}\left(I_{n}\right)$; we then say that $n \in \mathcal{N}^{0}$.

We prove

Lemma 3.1. For the limit (3.3) to hold $\omega$-almost surely, it suffices that, for any $n \in \mathcal{N}^{+}$, for $\varphi: \mathbb{R} \rightarrow \mathbb{R}^{+}$continuously differentiable and compactly supported, $\omega$-almost surely, one has

$$
\left|\mathcal{L}_{\omega, I_{n}, \Lambda}(\varphi)-\exp \left(-\int_{-\infty}^{+\infty}\left(1-e^{-\varphi(x)}\right) d x\right)\right|_{|\Lambda| \rightarrow+\infty}^{\rightarrow} 0 .
$$

Proof. As for $n \in \mathcal{N}^{0}, I_{n}$ is a single point, one computes

$$
\mathcal{L}_{\omega, \Lambda}(\varphi)=\sum_{n \in \mathcal{N}^{+}} \int_{I_{n}} e^{-\langle\Xi(\omega, t, \Lambda), \varphi\rangle} d t .
$$

Assume $J=[a, b]$. Fix $t \in I_{m}=\left(N\left(a_{m}\right), N\left(b_{m}\right)\right)$ for some $m \in \mathcal{N}^{+}$. For $m \in \mathcal{N}^{0}, N$ is constant equal to, say, $N_{m}$ on $I_{m}$. Assume that $\varphi$ has its support in $(-R, R)$. Then, for 
$|\Lambda|$ large (depending only on $R$ ), one computes

$$
\begin{aligned}
\langle\Xi(\omega, t, \Lambda), \varphi\rangle= & \left.\sum_{m \in \mathcal{N}^{0}} \#\left\{E_{n}(\omega, \Lambda) \in I_{m}\right\} \varphi\left(|\Lambda|\left[N_{n}-t\right)\right]\right) \\
& \left.+\sum_{m \in \mathcal{N}^{+}} \sum_{E_{n}(\omega, \Lambda) \in I_{m}} \varphi\left(|\Lambda|\left[N\left(E_{n}(\omega, \Lambda)\right)-t\right)\right]\right) \\
= & \left.\sum_{E_{n}(\omega, \Lambda) \in I_{m}} \varphi\left(|\Lambda|\left[N\left(E_{n}(\omega, \Lambda)\right)-t\right)\right]\right) \\
= & \left.\sum_{E_{n}(\omega, \Lambda) \in I_{m}} \varphi\left(\left|N\left(I_{m}\right)\right||\Lambda|\left[N_{I_{n}}\left(E_{n}(\omega, \Lambda)\right)-\left(t-N\left(a_{m}\right)\right) /\left|N\left(I_{m}\right)\right|\right)\right]\right) \\
= & \left\langle\Xi_{I_{m}}\left(\omega,\left(t-N\left(a_{m}\right)\right) /\left|N\left(I_{m}\right)\right|, \Lambda\right), \varphi\right\rangle
\end{aligned}
$$

On the other hand

$$
\int_{N\left(a_{m}\right)}^{N\left(b_{m}\right)} e^{-\left\langle\Xi_{I_{m}}\left(\omega,\left(t-N\left(a_{m}\right)\right) /\left|N\left(I_{m}\right)\right|, \Lambda\right), \varphi\right\rangle} d t=\left|N\left(I_{m}\right)\right| \int_{0}^{1} e^{-\left\langle\Xi_{I_{m}}(\omega, t, \Lambda), \varphi\right\rangle} d t .
$$

Recall that, as the measure defined by $N$ is absolutely continuous with respect to the Lebesgue measure, we have

$$
\sum_{n \in \mathcal{N}^{+}}\left|N\left(I_{m}\right)\right|=|N(J)|=1 .
$$

Thus, by Lebesgue's dominated convergence theorem, as $\mathcal{N}^{+}$is at most countable, we get that, if the necessary condition given in Lemma 3.1 is satisfied, then $\omega$-almost surely, we get

$$
\lim _{|\Lambda| \rightarrow+\infty} \mathcal{L}_{\omega, \Lambda}(\varphi)=\sum_{n \in \mathcal{N}^{+}}\left|N\left(I_{n}\right)\right| \lim _{|\Lambda| \rightarrow+\infty} \mathcal{L}_{\omega, I_{m}, \Lambda}(\varphi) .
$$

Thus, we have proved Lemma 3.1.

From now on, we assume that $N$ is a strictly increasing one-to-one mapping from $J$ to $[0,1]$ and prove Theorem 3.1 under this additional assumption.

Therefore, we first bring ourselves back to proving a similar result for "local" eigenvalues i.e. eigenvalues of restrictions of $H_{\omega}(\Lambda)$ to cubes much smaller than $\Lambda$ that lie inside small intervals i.e. much smaller than $J$. The "local" eigenvalues are those described by points (1), (2), (3) of Theorem 2.1. Using Lemma 2.1 then essentially brings ourselves back to the case of i.i.d. random variables uniformly distributed on $[0,1]$.

Theorem 2.1 does not give control on all the eigenvalues. To control the integral (3.1), this is not necessary: a good control of most of the eigenvalues is sufficient as Lemma 3.4 below shows. Theorem 2.2, which is a corollary of Theorem 2.1, is used to obtain good bounds on the number of controlled eigenvalues in the sense of Lemma 3.4.

3.2. Reduction to the study of local eigenvalues. Assume we are in the setting of Theorem 1.1 and that $N$ is as above i.e. $N$ is a strictly increasing Lipschitz continuous function from $J$ to $[0,1]$. Recall that $\nu$ is its derivative, the density of states.

To obtain our results, we will use Theorem 2.1 and Lemma 2.1. Therefore, we split the interval $I$ into small intervals and choose the length scale $\ell=\ell_{\Lambda}$ so that we can apply both Theorem 2.1 and Lemma 2.1 to these intervals. We now explain how this choice is done. 
Recall that $\rho$ is defined in (M) and pick $\rho^{\prime} \in(0, \rho /(1+(\rho+1) d))$. The computations done in [12, section 4.3.1] show that for any $\alpha \in(0,1)$ and $\nu \in(0,1 / d)$ such that

$$
0<1-\frac{1}{\alpha}+\frac{\rho-\rho^{\prime}}{1+\rho^{\prime}}-d \nu \rho,
$$

for $I_{\Lambda}$ (in the localization region) and $\ell=\ell_{\Lambda}$ such that

$$
\left|N\left(I_{\Lambda}\right)\right| \asymp|\Lambda|^{-\alpha} \text { and } \ell_{\Lambda} \asymp\left|N\left(I_{\Lambda}\right)\right|^{-\nu}
$$

if, in addition $I_{\Lambda}$ satisfies

$$
\left|N\left(I_{\Lambda}\right)\right| \geq\left|I_{\Lambda}\right|^{1+\rho^{\prime}}
$$

we can apply Theorem 2.1 and Lemma 2.1 to $I_{\Lambda}$ and

- (2.3) and (2.7) are satisfied;

- the right hand side in $(2.11)$ is $o\left(\left|N\left(I_{\Lambda}\right)\right||\Lambda|\right)$.

From now on we fix $\alpha \in(0,1), \nu \in(0,1 / d)$ and $\beta>0$ such that (3.6) be satisfied and, for later purposes, such that

$$
\frac{1}{\alpha}-1<\frac{\rho^{\prime}}{1+\rho^{\prime}}
$$

Partition $J=[a, b]$ into disjoint intervals $\left(J_{j, \Lambda}\right)_{1 \leq j \leq j_{\Lambda}}$ of weight $\left|N\left(J_{j, \Lambda}\right)\right|=|\Lambda|^{-\alpha}$ so that $j_{\Lambda}=|\Lambda|^{\alpha}$.

Define the sets

$$
B=\left\{1 \leq j \leq j_{\Lambda} ;\left|N\left(J_{j, \Lambda}\right)\right| \leq\left|J_{j, \Lambda}\right|^{1+\rho^{\prime}}\right\} \quad \text { and } \quad G=\left\{1, \cdots, j_{\Lambda}\right\} \backslash B .
$$

The set $B$ is the set of "bad" indices $j$ for which the interval $J_{j, \Lambda}$ does not satisfy the assumptions of Theorem 2.1, more precisely, does not satisfy the second condition in (3.8). For $j \in B$, one has

$$
\left|J_{j, \Lambda}\right| \geq\left|N\left(J_{j, \Lambda}\right)\right|^{1 /\left(1+\rho^{\prime}\right)}=|\Lambda|^{-\alpha /\left(1+\rho^{\prime}\right)} .
$$

Thus, one gets

$$
\# B \leq|\Lambda|^{\alpha /\left(1+\rho^{\prime}\right)}
$$

Fix $\alpha^{\prime} \in\left(\alpha, \min \left[1, \alpha\left(1+2 \rho^{\prime}\right) /\left(1+\rho^{\prime}\right)\right]\right)$. For $j \in G$, write $J_{j, \Lambda}=\left[a_{\Lambda}, b_{\Lambda}\right)$ and define

$$
K_{j, \Lambda}:=\left[a_{\Lambda}^{\prime}, b_{\Lambda}^{\prime}\right] \subset J_{j, \Lambda} \text { where }\left\{\begin{array}{l}
a_{\Lambda}^{\prime}=\inf \left\{a \geq a_{\Lambda} ; N(a)-N\left(a_{\Lambda}\right) \geq|\Lambda|^{-\alpha^{\prime}}\right\}, \\
b_{\Lambda}^{\prime}=\sup \left\{b \leq b_{\Lambda} ; N\left(b_{\Lambda}\right)-N(b) \geq|\Lambda|^{-\alpha^{\prime}}\right\} .
\end{array}\right.
$$

that is, $K_{j, \Lambda}$ is the interval $J_{j, \Lambda}$ where small neighborhoods of the endpoints have been remove.

Thus, our construction yields that

(1) the total density of states of the set we have remove is bounded by

$$
\sum_{j \in B}\left|N\left(J_{j, \Lambda}\right)\right|+\sum_{j \in G} N\left(J_{j, \Lambda} \backslash K_{j, \Lambda}\right) \lesssim|\Lambda|^{-\alpha+\alpha /\left(1+\rho^{\prime}\right)}+|\Lambda|^{-\alpha^{\prime}+\alpha} \lesssim|\Lambda|^{-\alpha \rho^{\prime} /\left(1+\rho^{\prime}\right)} ;
$$

(2) for $j \in G, t \in N\left(K_{j, \Lambda}\right)$ and $E \in J_{j^{\prime}, \Lambda}$ for $j^{\prime} \neq j$, one has

$$
|\Lambda||N(E)-t| \gtrsim|\Lambda|^{1-\alpha^{\prime}} \text {. }
$$


Note that one has

$$
|N(J)|=\sum_{j \in G}\left|N\left(J_{j, \Lambda}\right)\right|+\sum_{j \in B}\left|N\left(J_{j, \Lambda}\right)\right|=\sum_{j \in G}\left|N\left(K_{j, \Lambda}\right)\right|+O\left(|\Lambda|^{-\alpha \rho^{\prime} /\left(1+\rho^{\prime}\right)}\right) .
$$

Recall (3.2). Thus, for $\Lambda$ sufficiently large, by point (1) above, as $\varphi$ is non negative, one has

$$
\begin{aligned}
\int_{0}^{1} e^{-\langle\Xi(\omega, t, \Lambda), \varphi\rangle} d t & =\sum_{1 \leq j \leq G} \int_{N\left(K_{j, \Lambda}\right)} e^{-\langle\Xi(\omega, t, \Lambda), \varphi\rangle} d t+O\left(|\Lambda|^{-\alpha \rho^{\prime} /\left(1+\rho^{\prime}\right)}\right) \\
& =\sum_{1 \leq j \leq G} \int_{N\left(K_{j, \Lambda}\right)} e^{-\left\langle\Xi_{j}(\omega, t, \Lambda), \varphi\right\rangle} d t+O\left(|\Lambda|^{-\alpha \rho^{\prime} /\left(1+\rho^{\prime}\right)}\right)
\end{aligned}
$$

where, as $\varphi$ is compactly supported, by point (2) above, one has

$$
\left\langle\Xi_{j}(\omega, t, \Lambda), \varphi\right\rangle=\sum_{E_{n}(\omega, \Lambda) \in J_{j, \Lambda}} \varphi\left(|\Lambda|\left[N\left(E_{n}(\omega, \Lambda)\right)-t\right]\right) .
$$

Point (1) and (3.13) then yield

$$
\begin{aligned}
\int_{0}^{1} e^{-\langle\Xi(\omega, t, \Lambda), \varphi\rangle} d t & =\sum_{j \in G} \int_{N\left(J_{j, \Lambda}\right)} e^{-\left\langle\Xi_{j}(\omega, t, \Lambda), \varphi\right\rangle} d t+O\left(|\Lambda|^{-\alpha \rho^{\prime} /\left(1+\rho^{\prime}\right)}\right) \\
& =\sum_{j \in G}\left|N\left(J_{j, \Lambda}\right)\right| \int_{0}^{1} e^{-\left\langle\Xi_{J j, \Lambda}(\omega, t, \Lambda), \varphi\right\rangle} d t+O\left(|\Lambda|^{-\alpha \rho^{\prime} /\left(1+\rho^{\prime}\right)}\right)
\end{aligned}
$$

where $\Xi_{J_{j, \Lambda}}(\omega, t, \Lambda)$ is defined by (1.6) for $J=J_{j, \Lambda}$. Thus, following the proof of Lemma 3.1, the limit (3.4) will hold $\omega$-almost surely if we prove that, $\omega$ almost surely, one has

$$
\sup _{j \in G}\left|\int_{0}^{1} e^{-\left\langle\Xi_{J_{j, \Lambda}}(\omega, t, \Lambda), \varphi\right\rangle} d t-\exp \left(-\int_{-\infty}^{+\infty}\left(1-e^{-\varphi(x)}\right) d x\right)\right| \underset{|\Lambda| \rightarrow+\infty}{\rightarrow} 0 .
$$

Therefore, we first prove a weaker result, namely, almost sure convergence along a subsequence, that is

Lemma 3.2. Let $\Lambda_{L}$ be the cube of side length $L$ centered at 0 . Pick $\left(\alpha_{L}\right)_{L \geq 1}$ any sequence valued in $[1 / 2,2]$ such that $\alpha_{L} \rightarrow 1$ when $L \rightarrow+\infty$.

There exists $\nu>0$ such that, for $\varphi: \mathbb{R} \rightarrow \mathbb{R}^{+}$continuously differentiable and compactly supported, $\omega$-almost surely, one has

$$
\sup _{j \in G}\left|\int_{0}^{1} e^{-\left\langle\Xi_{J_{j, \Lambda_{L}}}\left(\omega, t, \Lambda_{L^{\nu}}\right), \varphi_{\alpha_{L}}\right\rangle} d t-\exp \left(-\int_{-\infty}^{+\infty}\left(1-e^{-\varphi(x)}\right) d x\right)\right| \underset{L \rightarrow+\infty}{\rightarrow} 0
$$

where, for $\alpha>0$, we have set, $\varphi_{\alpha}(\cdot)=\varphi(\alpha \cdot)$.

Indeed, Lemma 3.2, (3.14) and (3.13) clearly imply the claimed almost sure convergence on a subsequence; more precisely, it implies that, for $\left(\alpha_{L}\right)_{L \geq 1}$ a sequence such that $\alpha_{L} \rightarrow 1$ when $L \rightarrow+\infty$, $\omega$-almost surely,

$$
\left|\mathcal{L}_{\omega, \Lambda_{L^{\nu}}}\left(\varphi_{\alpha_{L}}\right)-\exp \left(-\int_{-\infty}^{+\infty}\left(1-e^{-\varphi(x)}\right) d x\right)\right| \underset{L \rightarrow+\infty}{\rightarrow} 0 .
$$

which is the claimed almost sure convergence on a subsequence for the choice of sequence $\alpha_{L}=1$.

To obtain the almost sure convergence on the whole sequence, we use 
Lemma 3.3. For some $\beta>0$, for $\varphi: \mathbb{R} \rightarrow \mathbb{R}^{+}$continuously differentiable and compactly supported, $\omega$-almost surely, for $L$ sufficiently large, one has

$$
\sup _{L^{\nu} \leq L^{\prime} \leq(L+1)^{\nu}}\left|\mathcal{L}_{\omega, \Lambda_{L^{\prime}}}(\varphi)-\mathcal{L}_{\omega, \Lambda_{L^{\nu}}}\left(\varphi_{\alpha_{L^{\prime}}}\right)\right| \lesssim L^{-\beta}
$$

where $\alpha_{L^{\prime}}=\left|\Lambda_{L^{\prime}}\right| /\left|\Lambda_{L^{\nu}}\right|$.

As $\alpha_{L} \rightarrow 1$ when $L \rightarrow+\infty$, equation (3.15) and, thus, Theorem 3.1, are immediate consequences of (3.17) and (3.18).

3.3. The proof of Lemma 3.2. The proof of Lemma 3.2 will consist in reducing the computation of the limit (3.16) to the case of i.i.d. random variables that have a distribution close to the uniform one. The number of these random variables will be random as well but large; it is controlled by Theorem 2.2.

We start with the statement and proof of a simple but useful result, namely,

Lemma 3.4. Pick a sequence of scale $\left(L_{p}\right)_{p \geq 1}$ such that $L_{p} \rightarrow+\infty$. For $p \geq 1$, consider two finite sequences $\left(x_{n}^{p}\right)_{1 \leq n \leq N_{p}}$ and $\left(y_{m}^{p}\right)_{1 \leq m \leq M_{p}}$ such that there exists $1 \leq K_{p} \leq$ $\inf \left(N_{p}, M_{p}\right)$ and sets $X_{p} \subset\left\{1, \cdots, N_{p}\right\}$ and $Y_{p} \subset\left\{1, \cdots, M_{p}\right\}$ s.t.

(1) $\# X_{p}=\# Y_{p}=K_{p}$ and $\left[\left(N_{p}-K_{p}\right)+\left(M_{p}-K_{p}\right)\right] / L_{p}=: a_{p} \rightarrow 0$,

(2) there exists a one-to-one map, say $\Psi_{p}: X_{p} \mapsto Y_{p}$ such that, for $n \in X_{p}$, one has $\left|x_{n}^{p}-y_{\Psi_{p}(n)}^{p}\right| \leq \varepsilon_{p} / L_{p}, \varepsilon_{p} \in[0,1]$

Fix $\alpha \in(0,1)$. Set $\Xi_{p}^{x}(t)=\sum_{n=1}^{N_{p}} \delta_{L_{p}\left[x_{n}^{p}-t\right]}$ and $\Xi_{p}^{y}(t)=\sum_{m=1}^{M_{p}} \delta_{L_{p}\left[y_{m}^{p}-t\right]}$. Then, for $p \geq 1$, one has

$$
\sup _{\varphi \in \mathcal{C}_{1, R}^{+}}\left|\int_{0}^{1} e^{-\left\langle\Xi_{p}^{x}(t), \varphi\right\rangle} d t-\int_{0}^{1} e^{-\left\langle\Xi_{p}^{y}(t), \varphi\right\rangle} d t\right| \leq 4 a_{p}^{\alpha}+e^{R \varepsilon_{p} K_{p}}-1 .
$$

where we have defined

$$
\mathcal{C}_{1, R}^{+}=\left\{\varphi: \mathbb{R} \rightarrow \mathbb{R}^{+} ; \quad \begin{array}{l}
\varphi \text { is continuously differentiable s.t. } \\
\operatorname{supp} \varphi \subset(-R, R) \text { and }\|\varphi\|_{\mathcal{C}^{1}} \leq R
\end{array}\right\}
$$

Proof of Lemma 3.4. Let $\tilde{X}_{p}=\left\{1, \cdots, N_{p}\right\} \backslash X_{p}$ and $\tilde{Y}_{p}=\left\{1, \cdots, M_{p}\right\} \backslash Y_{p}$. For $(n, m) \in$ $\tilde{X}_{p} \times \tilde{Y}_{p}$, define

$$
\begin{aligned}
I_{n}^{x} & =\left\{\begin{array}{l}
x_{n}^{p}+a_{p}^{\alpha}\left[N_{p}-K_{p}\right]^{-1}[-1,1] \text { if } \tilde{X}_{p} \neq \emptyset \text { i.e. } N_{p}-K_{p} \geq 1, \\
\emptyset \text { if not; }
\end{array}\right. \\
I_{m}^{y} & =\left\{\begin{array}{l}
y_{m}^{p}+a_{p}^{\alpha}\left[M_{p}-K_{p}\right]^{-1}[-1,1] \text { if } \tilde{Y}_{p} \neq \emptyset \text { i.e. } M_{p}-K_{p} \geq 1, \\
\emptyset \text { if not. }
\end{array}\right.
\end{aligned}
$$

Then, by point (1) of our assumptions on the sequences $\left(x_{n}^{p}\right)_{n}$ and $\left(y_{m}^{p}\right)_{m}$, one has

$$
\begin{aligned}
0 & \leq \int_{0}^{1} e^{-\left\langle\Xi_{p}^{x}(t), \varphi\right\rangle} d t-\int_{[0,1] \backslash\left[\left(\cup_{n \in \tilde{X}_{p}} I_{n}^{x}\right) \cup\left(\cup_{m \in \tilde{Y}_{p}} I_{m}^{y}\right]\right.} e^{-\left\langle\Xi_{p}^{x}(t), \varphi\right\rangle} d t \\
& \leq\left(N_{p}-K_{p}\right) a_{p}^{\alpha}\left[N_{p}-K_{p}\right]^{-1}+\left(M_{p}-K_{p}\right) a_{p}^{\alpha}\left[M_{p}-K_{p}\right]^{-1}=2 a_{p}^{\alpha}
\end{aligned}
$$


and, similarly

$$
0 \leq \int_{0}^{1} e^{-\left\langle\Xi_{p}^{y}(t), \varphi\right\rangle} d t-\int_{[0,1] \backslash\left[\left(\cup_{n \in \tilde{X}_{p}} I_{n}^{x}\right) \cup\left(\cup_{m \in \tilde{Y}_{p}} I_{m}^{y}\right]\right.} e^{-\left\langle\Xi_{p}^{y}(t), \varphi\right\rangle} d t \leq 2 a_{p}^{\alpha}
$$

On the other hand, for $t \in[0,1] \backslash\left[\left(\cup_{n \in \tilde{X}_{p}} I_{n}^{x}\right) \cup\left(\cup_{m \in \tilde{Y}_{p}} I_{m}^{y}\right]\right.$, one has

$$
L_{p} \operatorname{dist}\left(t, \tilde{X}_{p} \cup \tilde{Y}_{p}\right) \geq a_{p}^{\alpha} L_{p} \sup \left(\left[N_{p}-K_{p}\right]^{-1},\left[N_{p}-K_{p}\right]^{-1}\right) \geq a_{p}^{\alpha-1}>R
$$

for $p$ sufficiently large. Thus, for $t \in[0,1] \backslash\left[\left(\cup_{n \in \tilde{X}_{p}} I_{n}^{x}\right) \cup\left(\cup_{m \in \tilde{Y}_{p}} I_{m}^{y}\right]\right.$ and $\varphi \in \mathcal{C}_{1, R}^{+}$ (see $(3.20)$ ), one has

$$
\left\langle\Xi_{p}^{x}(t), \varphi\right\rangle=\sum_{n \in X_{p}} \varphi\left(L_{p}\left[x_{n}^{p}-t\right]\right) \quad \text { and } \quad\left\langle\Xi_{p}^{y}, \varphi\right\rangle=\sum_{m \in Y_{p}} \varphi\left(L_{p}\left[y_{m}^{p}-t\right]\right) .
$$

Now, by point (2) of our assumptions on the sequences $\left(x_{n}^{p}\right)_{n}$ and $\left(y_{m}^{p}\right)_{m}$, one has

$$
\sup _{\varphi \in \mathcal{C}_{1, R}^{+}} \sup _{\substack{t \in[0,1] \\ t \notin\left(\cup_{n \in \tilde{X}_{p}} I_{n}^{x}\right) \\ t \notin\left(\cup_{m \in \tilde{Y}_{p}}^{y} I_{m}^{y}\right)}}\left|\left\langle\Xi_{p}^{x}(t), \varphi\right\rangle-\left\langle\Xi_{p}^{y}(t), \varphi\right\rangle\right| \leq \varepsilon_{p} K_{p} \cdot \sup _{\varphi \in \mathcal{C}_{1, R}^{+}}\left\|\varphi^{\prime}\right\|_{\infty} \leq R \varepsilon_{p} K_{p} .
$$

Hence, as $\varphi$ is non negative, we obtain

$$
\sup _{\varphi \in \mathcal{C}_{1, R}^{+}}\left|\int_{[0,1] \backslash\left[\left(\cup_{n \in \tilde{X}_{p}} I_{n}^{x}\right) \cup\left(\cup_{n \in \tilde{Y}_{p}} I_{n}^{y}\right)\right]}\left(e^{-\left\langle\Xi_{p}^{x}(t), \varphi\right\rangle}-e^{-\left\langle\Xi_{p}^{y}(t), \varphi\right\rangle}\right) d t\right| \leq e^{R \varepsilon_{p} K_{p}}-1
$$

Combining (3.21), (3.22) and (3.24) completes the proof of Lemma 3.4.

Remark 3.1. Lemma 3.4, and, in particular, the error term coming from (3.23), can be improved if one assumes that the points in the sequences are not too densely packed. This is the case in the applications we have in mind. Though we do not use it here, it may be useful to treat the case of long range correlated random potentials where the error estimates of the local approximations of eigenvalues given by Theorem 2.1 can not be that precise anymore.

Fix $j \in G$ (see (3.10)). Pick $R$ large in Theorem 2.1. The construction done in the beginning of section 3.2 with the choice of scale $\ell_{\Lambda}$ given by (3.7) implies that one can apply

- Theorem 2.1 to the energy interval $I_{\Lambda}:=J_{j, \Lambda}$ for $H_{\omega}\left(\Lambda_{L}\right)$, the small cubes being of side length $\ell_{\Lambda}$;

- Theorem 2.1 and Lemma 2.1 to the energy interval $I_{\Lambda}:=J_{j, \Lambda}$ and any of the cubes $\Lambda_{\ell}(\gamma)$ of the decompostion obtained in Theorem 2.1.

Thus, we let $\mathcal{Z}_{\Lambda}^{j}$ be the set of configurations $\omega$ defined by Theorem 2.1 for the energy interval $I_{\Lambda}=J_{j, \Lambda}$. Then, (2.9) gives a lower bound on $\mathbb{P}\left(\mathcal{Z}_{\Lambda}^{j}\right)$ for any $p$ if $\Lambda$ is sufficiently large (see the comment following Theorem 2.1).

Let $\mathcal{N}_{\omega, j, \Lambda}^{b}$ be the set of indices $n$ of the eigenvalues $\left(E_{n}(\omega, \Lambda)\right)_{n}$ of $H_{\omega}(\Lambda)$ in $J_{j, \Lambda}$ that are not described by (1)-(3) of Theorem 2.1. Let $\mathcal{N}_{\omega, j, \Lambda}^{g}$ be the complementary set. Both sets are random. By (2.7) and our choice of lengthscales (see teh comment following Theorem 2.1), the number of eigenvalues not described by (1), (2) and (3) of Theorem 2.1, say, $N_{\omega, j, \Lambda}^{b}:=\# \mathcal{N}_{\omega, j, \Lambda}^{b}$ is bounded by, for some $\beta>0$,

$$
N_{\omega, j, \Lambda}^{b} \leq\left|N\left(J_{j, \Lambda}\right)\right||\Lambda|^{1-\beta}
$$


where as, by (2.11) in Theorem 2.2, the total number of eigenvalue of $H_{\omega}(\Lambda)$ in $J_{j, \Lambda}$, say, $N\left(J_{j, \Lambda}, \Lambda, \omega\right)$ satisfies, for some $\delta>0$, for any $p>0$ and $|\Lambda|$ sufficiently large,

$$
\mathbb{P}\left(\left|\frac{N\left(J_{j, \Lambda}, \Lambda, \omega\right)}{\left|N\left(J_{j, \Lambda}\right)\right||\Lambda|}-1\right| \geq|\Lambda|^{-\delta}\right) \leq|\Lambda|^{-p} .
$$

Let now $\mathcal{Z}_{\Lambda}^{j}$ be the set of configurations $\omega$ where one has both the conclusions of Theorem 2.1 and the bound

$$
\left|\frac{N\left(J_{j, \Lambda}, \Lambda, \omega\right)}{\left|N\left(J_{j, \Lambda}\right)\right||\Lambda|}-1\right| \leq|\Lambda|^{-\delta} .
$$

By (2.9) and (3.26), this new set still satisfies (2.9).

Define the following point measures:

- $\Xi_{J_{j, \Lambda}}^{g}(\omega, t, \Lambda):=\sum_{n \in \mathcal{N}_{\omega, j, \Lambda}^{g}} \delta_{\left|N\left(J_{j, \Lambda}\right)\right||\Lambda|\left[N_{J_{j, \Lambda}}\left(E_{n}(\omega, \Lambda)\right)-t\right]}$;

- for $\left(\Lambda_{\ell}\left(\gamma_{k}\right)\right)_{k}$, the cubes constructed in Theorem 2.1 (we write $\ell=\ell_{\Lambda}$ ), define the random variables:

$-X_{j, k}=X\left(\Lambda_{\ell}\left(\gamma_{k}\right), J_{j, \Lambda}\right)$ is the Bernoulli random variable

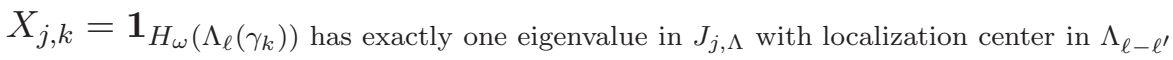

where $\ell=\ell_{\Lambda}$ and $\ell^{\prime}=\ell_{\Lambda}^{\prime}$ are chosen as described above;

- $\tilde{E}_{j, k}=\tilde{E}\left(\Lambda_{\ell}\left(\gamma_{k}\right), J_{j, \Lambda}\right)$ is this eigenvalue conditioned on the event $\left\{X_{j, k}=1\right\}$;

and the point measure $\Xi_{J_{j, \Lambda}}^{a p p}(\omega, t, \Lambda):=\sum_{k ; X_{j, k}=1} \delta_{\left|N\left(J_{j, \Lambda}\right)\right||\Lambda|\left[N_{J_{j, \Lambda}}\left(\tilde{E}_{j, k}\right)-t\right]}$.

We consider these point measures as random processes under the uniform distribution in $t$ in $[0,1]$.

We will need an estimate on the number

$$
N_{\omega, j, \Lambda}^{a p p}:=\left\{k ; X_{j, k}=1\right\} .
$$

It is provided by

Lemma 3.5. For any $p>0$, for $|\Lambda|$ sufficiently large, one has

$$
\mathbb{P}\left(\left|N_{\omega, j, \Lambda}^{a p p}-\right| N\left(J_{j, \Lambda}\right)|| \Lambda|| \geq\left[\left|N\left(J_{j, \Lambda}\right)\right||\Lambda|\right]^{2 / 3}\right) \leq e^{-\left[\left|N\left(J_{j, \Lambda}\right)\right||\Lambda|\right]^{1 / 3} / 3} \leq \mid \Lambda\left[\left.\right|^{-p} .\right.
$$

Proof. Lemma 3.5 follows by a standard large deviation argument for the i.i.d. Bernoulli random variables $\left(X_{j, k}\right)_{k}$ as, by Lemma 2.1 and our choice of $J_{j, \Lambda}$ and $\left(\ell^{\prime}, \ell\right)$ (for $\nu \in(\xi, 1)$ in Lemma 2.1), their common distribution satisfies

$$
P\left(X_{j, k}=1\right)=\left|N\left(J_{j, \Lambda}\right)\right|\left|\Lambda_{\ell}\right|(1+o(1)) .
$$

The proof of Lemma 3.5 is complete.

Thus, one may restrict once more the set of configurations $\omega$ to those such that, for some $\delta>0$

$$
\left|\frac{N_{\omega, j, \Lambda}^{a p p}}{\left|N\left(J_{j, \Lambda}\right)\right||\Lambda|}-1\right| \leq|\Lambda|^{-\delta} .
$$

and call this set again $\mathcal{Z}_{\Lambda}^{j}$. By Lemma 3.5 and (2.9), the probability of this set also satisfies (2.9) for any $p>0$ provided $|\Lambda|$ is sufficiently large.

Using Lemma 3.4, one then proves 
Lemma 3.6. For some $\beta>0$, for $\omega \in \mathcal{Z}_{\Lambda}^{j}$ and $\Lambda$ sufficiently large, one has,

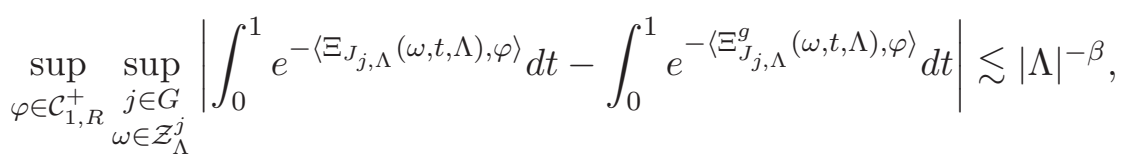

and

$$
\sup _{\varphi \in \mathcal{C}_{1, R}^{+}} \sup _{\substack{j \in G \\ \omega \in \mathcal{Z}_{\Lambda}^{j}}}\left|\int_{0}^{1} e^{-\left\langle\Xi_{J_{j, \Lambda}^{g}}^{g}(\omega, t, \Lambda), \varphi\right\rangle} d t-\int_{0}^{1} e^{-\left\langle\Xi_{J_{j, \Lambda}}^{a p p}(\omega, t, \Lambda), \varphi\right\rangle} d t\right| \lesssim|\Lambda|^{-\beta} .
$$

The proof of Lemma 3.6. As underlined above, the statements of Lemma 3.6 are corollaries of Lemma 3.4.

To obtain (3.30), for $p=|\Lambda|$, it suffices to take

- $x_{n}^{p}=E_{n}(\omega, \Lambda)$ for $1 \leq n \leq N\left(J_{j, \Lambda}, \Lambda, \omega\right)$,

- $y_{n}^{p}=E_{n}(\omega, \Lambda)$ for $n \in \mathcal{N}_{\omega, j, \Lambda}^{g}$.

Assumption (2) in Lemma 3.4 is clearly fulfilled as $\left(x_{n}^{p}\right)_{n}$ is a subsequence of $\left(y_{n}^{p}\right)_{n}$. Assumption (1) is an immediate consequence (3.25) and (3.27).

Let us now prove (3.31). Notice that, by Theorem 2.1, one has $N_{\omega, j, \Lambda}^{a p p} \geq N_{\omega, j, \Lambda}^{g}$. Moreover, to each $n \in \mathcal{N}_{\omega, j, \Lambda}^{g}$, one can associate a unique $1 \leq k(n) \leq N_{\omega, j, \Lambda}^{a p p}$ such that $X_{j, k(n)}=1$ and the first part of (2.5) hold.

To prove (3.31), for $p=|\Lambda|$, it suffices to set

- $x_{n}^{p}=\tilde{E}_{j, k(n)}$ for $k(n)$ such that $X_{j, k(n)}=1$,

- $y_{n}^{p}=E_{n}(\omega, \Lambda)$ for $n \in \mathcal{N}_{\omega, j, \Lambda}^{g}$.

So we may take $K_{p}=N_{\omega, j, \Lambda}^{g}$. By the first part of (2.5), we know that assumption (2) of Lemma 3.4 is satisfied with $\varepsilon_{p}=|\Lambda|^{-2}$. Thus, $\varepsilon_{p} \cdot K_{p} \lesssim|\Lambda|^{-1}$.

That assumption (1) is satisfied follows immediately from (3.25) and (3.29).

This completes the proof of Lemma 3.6

So we have reduced the problem to analyzing the case of i.i.d. random variables. In the next sections, we prove

Lemma 3.7. Fix $\rho^{\prime} \in(0, \rho /(1+\rho))$ where $\rho$ is defined by $(M)$. Fix $\alpha \in(0,1)$ and $\nu \in(0,1)$ satisfying (3.6) and (3.9). There exists $\kappa>1 / d$ such that, for any $\left(\alpha_{L}\right)_{L \geq 1}$ a sequence valued in $[1 / 2,2]$, one has

$\sum_{j \in G} \sum_{L \geq 1} \mathbb{E}\left(\left[\int_{0}^{1} e^{-\left\langle\Xi_{J_{j, \Lambda^{\kappa}}}^{a p p}\left(\omega, t, \Lambda_{L^{\kappa}}\right), \varphi_{\alpha_{L}}\right\rangle} d t-\exp \left(-\kappa_{L} \int_{-\infty}^{+\infty}\left(1-e^{-\varphi_{\alpha_{L}}(x)}\right) d x\right)\right]^{2}\right)<+\infty$.

Let us now complete the proof of Lemma 3.2 using Lemmas 3.6 and 3.7 and (2.9) the estimates on the probability of $\mathcal{Z}_{\Lambda}^{j}$.

Clearly, Lemma 3.7 implies that

$$
\mathbb{E}\left(\limsup _{L \geq 1} \sup _{j \in G}\left|\int_{0}^{1} e^{-\left\langle\Xi\left(\omega, t, j, \Lambda_{L^{\kappa}}\right), \varphi_{\alpha_{L}}\right\rangle} d t-\exp \left(-\kappa_{L} \int_{-\infty}^{+\infty}\left(1-e^{-\varphi_{\alpha_{L}}(x)}\right) d x\right)\right|\right)=0 .
$$

As all the integrands are bounded by 1, by (2.9), (3.30) and (3.31), we know that

$$
\mathbb{E}\left(\limsup _{L \geq 1} \sup _{j \in G}\left|\int_{0}^{1} e^{-\left\langle\Xi_{J_{j, \Lambda_{L}}}\left(\omega, t, \Lambda_{L^{\kappa}}\right), \varphi_{\alpha_{L}}\right\rangle} d t-\int_{0}^{1} e^{-\left\langle\Xi_{J_{j, \Lambda_{L}}}^{g}\left(\omega, t, \Lambda_{L^{\kappa}}\right), \varphi_{\alpha_{L}}\right\rangle} d t\right|\right)=0
$$


and

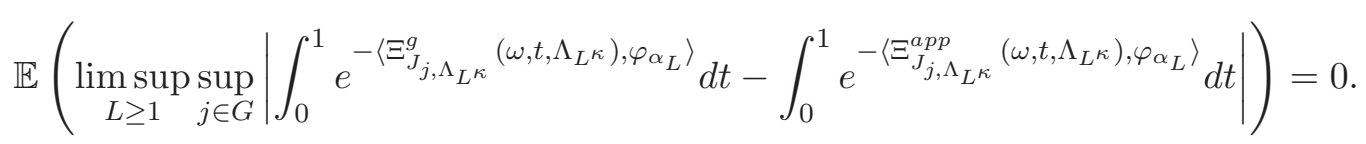

Thus, if $\alpha_{L} \rightarrow 1$ when $L \rightarrow+\infty$, one has

$$
\exp \left(-\kappa_{L} \int_{-\infty}^{+\infty}\left(1-e^{-\varphi_{\alpha_{L}}(x)}\right) d x\right) \underset{L \rightarrow+\infty}{\rightarrow} \exp \left(-\int_{-\infty}^{+\infty}\left(1-e^{-\varphi(x)}\right) d x\right)
$$

this clearly implies (3.16) and completes the proof of Lemma 3.2.

3.4. The proof of Lemma 3.7. Let us recall a few facts that will be of use in this proof. Write $\Lambda_{\ell}=\Lambda_{\ell}(0)$ and define the random variables $X$ and $\tilde{E}$ as in the beginning of section 2.1 for $I_{\Lambda}=J_{j, \Lambda}$ and the cube $\Lambda_{\ell}$. Recall that the cube $\Lambda=\Lambda_{L}$ is much larger than $\Lambda_{\ell}$. Now, pick $N_{\omega, j, \Lambda}^{a p p}$ independent copies of $\tilde{E}$, say $\left(\tilde{E}_{k}\right)_{1 \leq k \leq N_{\omega, j, \Lambda}^{a p p}}$. Then, the random process $\Xi_{J_{j, \Lambda}}^{a p p}$ is the process

$$
\Xi_{J_{j, \Lambda}}^{a p p}(\omega, t, \Lambda):=\sum_{1 \leq k \leq N_{\omega, j, \Lambda}^{a p p}} \delta_{\left|N\left(J_{j, \Lambda}\right)\right||\Lambda|\left[N_{J_{j, \Lambda}}\left(\tilde{E}_{k}\right)-t\right]} .
$$

By Lemma 3.4 and (3.29), it thus suffices to study the point process

$$
\Xi(\omega, t, j, \Lambda):=\sum_{1 \leq k \leq|\Lambda|\left|N\left(J_{j, \Lambda}\right)\right|} \delta_{\left|N\left(J_{j, \Lambda}\right)\right||\Lambda|\left[N_{J_{j, \Lambda}}\left(\tilde{E}_{k}\right)-t\right]} .
$$

Recall that $N_{J_{j, \Lambda}}$ is defined by (1.7) for $J=J_{j, \Lambda}$. Pick $\varphi \in \mathcal{C}_{1, R}^{+}$(see (3.20)). As the random variables $\left(\tilde{E}_{k}\right)_{1 \leq k \leq\left|N\left(J_{j, \Lambda}\right)\right||\Lambda|}$ are i.i.d., one computes

$$
\mathbb{E}\left(\int_{0}^{1} e^{-\langle\Xi(\omega, t, j, \Lambda), \varphi\rangle} d t\right)=\int_{0}^{1} \Phi\left(t, \Lambda, J_{j, \Lambda}, \varphi\right) d t
$$

and

$$
\mathbb{E}\left(\left[\int_{0}^{1} e^{-\langle\Xi(\omega, t, j, \Lambda), \varphi\rangle} d t\right]^{2}\right)=\int_{0}^{1} \int_{0}^{1} \Phi\left(t, t^{\prime}, \Lambda, J_{j, \Lambda}, \varphi\right) d t d t^{\prime}
$$

where

$$
\Phi\left(t, \Lambda, J_{j, \Lambda}, \varphi\right)=\left[1-\mathbb{E}\left(1-e^{-\varphi\left(\left|N\left(J_{j, \Lambda}\right)\right||\Lambda|\left[N_{J_{j, \Lambda}}(\tilde{E})-t\right]\right)}\right)\right]^{\left|N\left(J_{j, \Lambda}\right)\right||\Lambda|}
$$

and

$$
\begin{aligned}
& \Phi\left(t, t^{\prime}, \Lambda, J_{j, \Lambda}, \varphi\right) \\
& =\left[1-\mathbb{E}\left(1-e^{-\varphi\left(\left|N\left(J_{j, \Lambda}\right)\right||\Lambda|\left[N_{J_{j, \Lambda}}(\tilde{E})-t\right]\right)-\varphi\left(\left|N\left(J_{j, \Lambda}\right)\right||\Lambda|\left[N_{J_{j, \Lambda}}(\tilde{E})-t^{\prime}\right]\right)}\right)\right]^{\left|N\left(J_{j, \Lambda}\right)\right||\Lambda|} .
\end{aligned}
$$

If $E \mapsto N_{J_{j, \Lambda}}(E)$ were the distribution function of the random variable $\tilde{E}$, the random variables $N_{J_{j, \Lambda}}(\tilde{E})$ would be distributed uniformly on $[0,1]$ and the desired result would be standard and follow e.g. from the computations done in the appendix of [25]. The distribution function of $\tilde{E}$ is described by Lemma 2.1. As we only consider $j \in G$, we know that $\left|N\left(J_{j, \Lambda}\right)\right| \geq\left|J_{j, \Lambda}\right|^{1+\rho^{\prime}}$ for some $\rho^{\prime} \in(0, \rho /(1+(d+1) \rho))$. Thus, choosing $\nu \in(\xi, 1)$ in 
Lemma 2.1, for $x \in J_{j, \Lambda}$ (take $y=0$ ), using (2.1) and (3.6), the estimation (2.2) becomes, for any $p>0$ and $|\Lambda|=\left|\Lambda_{L}\right|$ sufficiently large,

$$
\begin{aligned}
\left|\kappa_{\Lambda} \cdot\right| N\left(J_{j, \Lambda}\right)|| \Lambda|\tilde{\Xi}(x)-| N\left(J_{j, \Lambda}\right)|| \Lambda\left|N_{J_{j, \Lambda}}(x)\right| & \lesssim\left|N\left(J_{j, \Lambda}\right)\right||\Lambda|\left[\left|J_{j, \Lambda}\right|\left|\Lambda_{\ell}\right|\right]^{\rho} \\
& \lesssim\left|N\left(J_{j, \Lambda}\right)\right|^{1-\alpha^{-1}-d \nu \rho+\rho /(1+\tilde{\rho})} \\
& \lesssim\left|N\left(J_{j, \Lambda}\right)\right|^{\rho^{\prime} /\left(1+\rho^{\prime}\right)}
\end{aligned}
$$

where, by (2.1) and the same computation as in (3.37), one has, for some $\beta>0$,

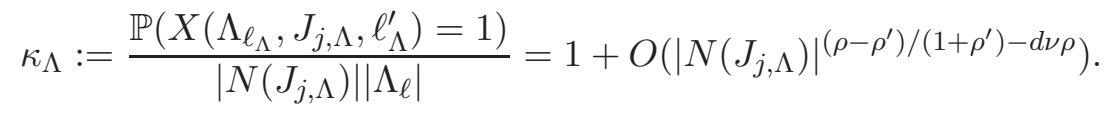

Using (3.37), from (3.35), as $\varphi \in \mathcal{C}_{1, R}^{+}$, we derive

$$
\begin{aligned}
& \frac{\log \Phi\left(t, \Lambda, J_{j, \Lambda}, \varphi\right)}{\left|N\left(J_{j, \Lambda}\right)\right||\Lambda|} \\
& \quad=\log \left[1-\mathbb{E}\left(1-e^{-\varphi\left(\left|N\left(J_{j, \Lambda}\right)\right||\Lambda|\left[\kappa_{\Lambda} \cdot \tilde{\Xi}(\tilde{E})-t\right]\right)}\right)+O\left(\left|N\left(J_{j, \Lambda}\right)\right|^{\rho^{\prime} /\left(1+\rho^{\prime}\right)}\right)\right] .
\end{aligned}
$$

Now, fix $\kappa \in(0,1)$. The random variable $\tilde{\Xi}(\tilde{E})$ is uniformly distributed on $[0,1]$; thus, we compute

$$
\begin{aligned}
\mathbb{E}\left(1-e^{-\varphi\left(\left|N\left(J_{j, \Lambda}\right)\right||\Lambda|\left[\kappa_{\Lambda} \cdot \tilde{\Xi}(\tilde{E})-t\right]\right)}\right)=\int_{0}^{1}\left(1-e^{-\varphi\left(\left|N\left(J_{j, \Lambda}\right)\right||\Lambda|\left[\kappa_{\Lambda} u-t\right]\right)}\right) d u \\
=\frac{1}{\kappa_{\Lambda}\left|N\left(J_{j, \Lambda}\right)\right||\Lambda|} \int_{-\left|N\left(J_{j, \Lambda}\right)\right||\Lambda| t}^{\left|N\left(J_{j, \Lambda}\right)\right||\Lambda|\left[\kappa_{\Lambda}-t\right]}\left(1-e^{-\varphi(u)}\right) d u \\
=\frac{1}{\kappa_{\Lambda}\left|N\left(J_{j, \Lambda}\right)\right||\Lambda|} \int_{-\infty}^{+\infty}\left(1-e^{-\varphi(u)}\right) d u
\end{aligned}
$$

if, using (3.7), we assume that $t$ satisfies

$$
\left|N\left(J_{j, \Lambda}\right)\right|^{\left(\alpha^{-1}-1\right) \kappa} \leq t \leq 1-\left|N\left(J_{j, \Lambda}\right)\right|^{\left(\alpha^{-1}-1\right) \kappa}+O\left(\left|N\left(J_{j, \Lambda}\right)\right|^{\left(\rho-\rho^{\prime}\right) /\left(1+\rho^{\prime}\right)-d \nu \rho}\right)
$$

for $|\Lambda|$ sufficiently large (as $\alpha \in(0,1)$ and $\left|N\left(J_{j, \Lambda}\right)\right| \rightarrow 0$ when $|\Lambda| \rightarrow+\infty$ ). Here, we have used (3.38).

Now, if we take $\alpha$ in (3.7) so small that (3.9) be satisfied then, (3.39) and (3.40) yield that, for any $\beta \in\left(0, \rho^{\prime} /\left(1+\rho^{\prime}\right)\right)$, for $t$ satisfying (3.41) and $|\Lambda|$ sufficiently large,

$$
\log \Phi\left(t, \Lambda, J_{j, \Lambda}, \varphi\right)=\frac{1}{\kappa_{\Lambda}} \int_{-\infty}^{+\infty}\left(1-e^{-\varphi(u)}\right) d u+O\left(\left|N\left(J_{j, \Lambda}\right)\right|^{\beta}\right) .
$$

Thus, by (3.38), for $\beta \in\left(0, \min \left(\rho^{\prime}, \rho-\rho^{\prime}\right) /\left(1+\rho^{\prime}\right)\right)$, for $t$ satisfying (3.41) and $|\Lambda|$ sufficiently large,

$$
\log \Phi\left(t, \Lambda, J_{j, \Lambda}, \varphi\right)=\int_{-\infty}^{+\infty}\left(1-e^{-\varphi(u)}\right) d u+O\left(\left|N\left(J_{j, \Lambda}\right)\right|^{\beta}\right) .
$$

Using (3.37), from (3.36), as $\varphi \in \mathcal{C}_{1, R}^{+}$, we derive

$$
\begin{aligned}
& \frac{\log \Phi\left(t, t^{\prime}, \Lambda, J_{j, \Lambda}, \varphi\right)}{\left|N\left(J_{j, \Lambda}\right)\right||\Lambda|} \\
& \quad=\log \left[1-\mathbb{E}\left(1-e^{-\varphi\left(\left|N\left(J_{j, \Lambda}\right)\right| \mid\left[\kappa_{\Lambda} \cdot \tilde{\Xi}(\tilde{E})-t\right]\right)-\varphi\left(\left|N\left(J_{j, \Lambda}\right)\right||\Lambda|\left[\kappa_{\Lambda} \tilde{\Xi}(\tilde{E})-t^{\prime}\right]\right)}\right)\right. \\
& \left.+O\left(\left|N\left(J_{j, \Lambda}\right)\right|^{\rho^{\prime} /\left(1+\rho^{\prime}\right)}\right)\right] .
\end{aligned}
$$


Moreover, for $t$ and ' $t$ satisfying (3.41) such that

$$
\left|N\left(J_{j, \Lambda}\right)\right|^{\left(\alpha^{-1}-1\right) \kappa} \leq\left|t-t^{\prime}\right|
$$

as above, one computes

$$
\begin{aligned}
\mathbb{E}\left(1-e^{-\varphi\left(\left|N\left(J_{j, \Lambda}\right)\right| \mid\left[\kappa_{\Lambda} \cdot \tilde{\Xi}(\tilde{E})-t\right]\right)-\varphi\left(\left|N\left(J_{j, \Lambda}\right)\right||\Lambda|\left[\kappa_{\Lambda} \tilde{\Xi}(\tilde{E})-t^{\prime}\right]\right)}\right) \\
\quad=\int_{0}^{1}\left(1-e^{-\varphi\left(\left|N\left(J_{j, \Lambda}\right)\right||\Lambda|\left[\kappa_{\Lambda} u-t\right]\right)-\varphi\left(\left|N\left(J_{j, \Lambda}\right)\right||\Lambda|\left[\kappa_{\Lambda} u-t^{\prime}\right]\right)}\right) d u \\
=\frac{2}{\kappa_{\Lambda}\left|N\left(J_{j, \Lambda}\right)\right||\Lambda|} \int_{-\infty}^{+\infty}\left(1-e^{-\varphi(u)}\right) d u
\end{aligned}
$$

for $|\Lambda|$ sufficiently large. Here, we have used (3.38).

Again, if we take $\alpha$ in (3.7) so small that (3.9) is satisfied then, (3.42), (3.38) and (3.44) yield that, for any $\beta \in\left(0, \min \left(\rho^{\prime}, \rho-\rho^{\prime}\right) /\left(1+\rho^{\prime}\right)\right)$, for $t$ and ' $t$ satisfying (3.41) and (3.43), for $|\Lambda|$ sufficiently large,

$$
\log \Phi\left(t, t^{\prime}, \Lambda, J_{j, \Lambda}, \varphi\right)=2 \int_{-\infty}^{+\infty}\left(1-e^{-\varphi(u)}\right) d u+O\left(\left|N\left(J_{j, \Lambda}\right)\right|^{\beta}\right)
$$

Finally notice that $\Phi\left(t, \Lambda, J_{j, \Lambda}, \varphi\right)$ and $\Phi\left(t, t^{\prime}, \Lambda, J_{j, \Lambda}, \varphi\right)$ are both bounded by 1 and that the measure of the sets of $t \in[0,1]$ satisfying (3.41) and the measure of the sets of $\left(t, t^{\prime}\right) \in[0,1]^{2}$ satisfying (3.41) for $t$ and $t^{\prime}$ and (3.43) are both larger than

$$
1-O\left(\left|N\left(J_{j, \Lambda}\right)\right|^{\left(\alpha^{-1}-1\right) \kappa}\right)+O\left(\left|N\left(J_{j, \Lambda}\right)\right|^{\left(\rho-\rho^{\prime}\right) /\left(1+\rho^{\prime}\right)-d \nu \rho}\right) .
$$

Thus, thus taking into account (3.7), we have proved

Lemma 3.8. Fix $R>0$. Fix $\rho^{\prime} \in(0, \rho /(1+\rho))$ where $\rho$ is defined by $(M)$. Fix $\alpha \in(0,1)$ and $\nu \in(0,1)$ satisfying $(3.6)$ and $(3.9)$.

There exists $\beta>0$ such that, for $|\Lambda|$ sufficiently large (depending only on $R, \rho^{\prime}, \alpha$ and $\nu$ ), one has

$$
\sup _{\varphi \in \mathcal{C}_{1, R}^{+}} \sup _{j \in G}\left|\int_{0}^{1} \Phi\left(t, \Lambda, J_{j, \Lambda}, \varphi\right) d t-\exp \left(-\int_{-\infty}^{+\infty}\left(1-e^{-\varphi(x)}\right) d x\right)\right| \leq|\Lambda|^{-\beta}
$$

and

$$
\begin{aligned}
\sup _{\varphi \in \mathcal{C}_{1, R}^{+}} \sup _{j \in G}\left|\int_{0}^{1} \int_{0}^{1} \Phi\left(t, t^{\prime}, \Lambda, J_{j, \Lambda}, \varphi\right) d t d t^{\prime}-\exp \left(-2 \int_{-\infty}^{+\infty}\left(1-e^{-\varphi(x)}\right) d x\right)\right| \\
\leq|\Lambda|^{-\beta} .
\end{aligned}
$$

Let us use Lemma 3.8 to complete the proof of Lemma 3.7. For $L \geq 1$, let $\Lambda=\Lambda_{L}$. Fix $\left(\alpha_{L}\right)_{L \geq 1}$ a sequence valued in $[1 / 2,2]$. Then, for $\varphi \in \mathcal{C}_{1, R}^{+}$, the sequence $\left(\varphi_{\alpha_{L}}\right)_{L \geq 1}$ is bounded in $\mathcal{C}_{1,2 R}^{+}$. Thus, by Lemma 3.8, for $\kappa$ such that $\kappa \beta d>1$ and $\left(\alpha_{L}\right)_{L \geq 1}$, any sequence valued in $[1 / 2,2]$, we have that

$$
\sum_{j \in G} \sum_{L \geq 1} \mathbb{E}\left(\left[\int_{0}^{1} e^{-\left\langle\Xi\left(\omega, t, j, \Lambda_{L^{\kappa}}\right), \varphi_{\alpha_{L}}\right\rangle} d t-\exp \left(-\int_{-\infty}^{+\infty}\left(1-e^{-\varphi_{\alpha_{L}}(x)}\right) d x\right)\right]^{2}\right)<+\infty .
$$

Thus, if $\alpha_{L} \rightarrow 1$ as $L \rightarrow+\infty$, we have proved Lemma 3.7. 
3.5. The proof of Lemma 3.3. Clearly, by (3.13) and (3.14), to prove Lemma 3.3, it suffices to show that, for some $\beta>0$, $\omega$-almost surely, one has

$$
\sup _{\substack{j \in G \\ L^{\kappa} \leq L^{\prime} \leq(L+1)^{\kappa}}}\left|\int_{0}^{1} e^{-\left\langle\Xi_{J_{j, \Lambda_{L^{\kappa}}}}\left(\omega, t, \Lambda_{L^{\prime}}\right), \varphi\right\rangle} d t-\int_{0}^{1} e^{-\left\langle\Xi_{J_{j, \Lambda_{L^{\kappa}}}}\left(\omega, t, \Lambda_{L^{\kappa}}\right), \varphi_{\alpha_{L^{\prime}}}\right\rangle} d t\right| \lesssim L^{-\beta}
$$

where $\alpha_{L^{\prime}}=\left|\Lambda_{L^{\prime}}\right| /\left|\Lambda_{L^{k}}\right|$. Notice here that we chose the same partition of $J$ into $\left(J_{j, \Lambda_{L^{\kappa}}}\right)_{j}$ for all $L^{\kappa} \leq L^{\prime} \leq(L+1)^{\kappa}$ which is possible as $\left|\Lambda_{L^{\prime}}\right|=\left|\Lambda_{L^{\nu}}\right|(1+o(1))$.

For $\Lambda^{\prime} \subset \Lambda$, let $E_{1}\left(\omega, \Lambda, \Lambda^{\prime}\right) \leq E_{2}\left(\omega, \Lambda, \Lambda^{\prime}\right) \leq \cdots \leq E_{N\left(J, \Lambda, \Lambda^{\prime}, \omega\right)}\left(\omega, \Lambda, \Lambda^{\prime}\right)$ be the eigenvalues of $H_{\omega}(\Lambda)$ in $J$ with localization center in $\Lambda^{\prime}$, and, thus, $N\left(J, \Lambda, \Lambda^{\prime}, \omega\right)$ be their number which is random. Recall that $N(J, \Lambda, \omega)=N(J, \Lambda, \Lambda, \omega)$ denotes the number of eigenvalues of $H_{\omega}(\Lambda)$ in $J$.

In Lemma 3.9, we prove that most eigenvalues of $H_{\omega}\left(\Lambda_{L^{\prime}}\right)$ and of $H_{\omega}\left(\Lambda_{L^{\nu}}\right)$ in $J$ have center of localization in $\Lambda_{(L-1)^{\nu}}$; this is essentially a consequence of the description given by Theorem 2.1. Thus, by Lemma 2.2, these eigenvalues of $H_{\omega}\left(\Lambda_{L^{\prime}}\right)$ and of $H_{\omega}\left(\Lambda_{L^{\nu}}\right)$ are close to one another. We can then use Lemma 3.4 to compare $\Xi_{J_{j, \Lambda_{L^{\kappa}}}}\left(\omega, t, \Lambda_{L^{\prime}}\right)$ and $\Xi_{J_{j, \Lambda_{L^{\kappa}}}}\left(\omega, t, \Lambda_{L^{\kappa}}\right)$.

We prove

Lemma 3.9. Pick $\nu>0$. There exists $\beta>0$ such that, $\omega$-almost surely, for $L$ sufficiently large and $L^{\kappa} \leq L^{\prime} \leq(L+1)^{\kappa}$ and $j \in G$, one has

$$
\left|\frac{N\left(J_{j, \Lambda_{L^{\kappa}}}, \Lambda_{L^{\prime}}, \Lambda_{(L-1)^{\nu}}, \omega\right)}{N\left(J_{j, \Lambda_{L^{\kappa}}}, \Lambda_{L^{\kappa}}, \Lambda_{(L-1)^{\nu}}, \omega\right)}-1\right|+\left|\frac{N\left(J_{j, \Lambda_{L^{\kappa}}}, \Lambda_{L^{\prime}}, \Lambda_{(L-1)^{\nu}}, \omega\right)}{N\left(J_{j, \Lambda_{L^{\kappa}}}, \Lambda_{L^{\prime}}, \omega\right)}-1\right| \lesssim L^{-\beta} ;
$$

$$
\left|\frac{N\left(K_{j, \Lambda_{L^{\kappa}}}, \Lambda_{L^{\prime}}, \omega\right)}{N\left(J_{j, \Lambda_{L^{\kappa}}}, \Lambda_{L^{\prime}}, \omega\right)}-1\right|+\left|\frac{N\left(K_{j, \Lambda_{L^{\kappa}}}, \Lambda_{L^{\kappa}}, \omega\right)}{N\left(J_{j, \Lambda_{L^{\kappa}}}, \Lambda_{L^{\kappa}}, \omega\right)}-1\right| \lesssim L^{-\beta}
$$

where $\left(K_{j, \Lambda}\right)_{j}$ are defined in the beginning of section 3.2;

(3) to each eigenvalue of $H_{\omega}\left(\Lambda_{L^{\prime}}\right)$ in $K_{j, \Lambda_{L^{\kappa}}}$ with localization center in $\Lambda_{(L-1)^{\nu}}$, say, $E$, one can associate an eigenvalue of $H_{\omega}\left(\Lambda_{L^{\kappa}}\right)$ in $J_{j, \Lambda_{L^{\kappa}}}$, say, $E^{\prime}$, such that $\left|E-E^{\prime}\right| \leq$ $L^{-3 d \nu}$

(4) to each eigenvalue of $H_{\omega}\left(\Lambda_{L^{\kappa}}\right)$ in $J$ with localization center in $\Lambda_{(L-1)^{\nu}}$ in $K_{j, \Lambda_{L^{\kappa}}}$, say, $E$, one can associate an eigenvalue of $H_{\omega}\left(\Lambda_{L^{\prime}}\right)$ in $J_{j, \Lambda_{L^{k}}}$, say, $E^{\prime}$, such that $\left|E-E^{\prime}\right| \leq L^{-3 d \nu}$.

We now can apply Lemma 3.7 to $\left\langle\Xi_{J_{j, \Lambda_{L^{\kappa}}}}\left(\omega, t, \Lambda_{L^{\prime}}\right), \varphi\right\rangle$ and $\left\langle\Xi_{J_{j, \Lambda_{L^{\kappa}}}}\left(\omega, t, \Lambda_{L^{\kappa}}\right), \varphi_{\alpha_{L}^{\prime}}\right\rangle$. By Lemma 3.9, the assumptions of Lemma 3.7 will be satisfied if, using the notations of Lemma 3.7, we take

- $X_{p}$ to be the eigenvalues of $H_{\omega}\left(\Lambda_{L^{\prime}}\right)$ in $K_{j, \Lambda_{L^{\kappa}}}$ with localization center in $\Lambda_{(L-1)^{\nu}}$,

- $Y_{p}$ to be the eigenvalues of $H_{\omega}\left(\Lambda_{L^{\kappa}}\right)$ in $K_{j, \Lambda_{L^{\kappa}}}$ with localization center in $\Lambda_{(L-1)^{\nu}}$.

Indeed, Lemma 3.9 then provides the estimates

$$
0 \leq a_{p} \lesssim L^{-\beta}, \quad 0 \leq K_{p} \leq C L^{d \nu+1} \quad \text { and } \quad 0 \leq \varepsilon_{p} \leq L^{-3 d \nu} .
$$

Then, (3.18) and, thus, Lemma 3.3, is an immediate consequence of Lemma 3.7 (where one of the functions $\varphi$ has been replaced with $\left.\varphi_{\alpha_{L^{\prime}}}\right)$. This completes the proof of Lemma 3.3. 
Proof of Lemma 3.9. First, in Theorem 2.1 (see the proofs in [12] for more details), for $L^{\kappa} \leq L^{\prime} \leq(L+1)^{\nu}$, one can pick the same scale $\ell$. Then, by Theorem 2.1 (for $R>2 \nu$ ), for any $p>0$ and some $\beta>0$, we know that, with a probability at least $1-L^{-p}$, for $L^{\kappa} \leq L^{\prime} \leq$ $(L+1)^{\nu}$ and $j \in G$ (recall that $\left.\# G \leq\left|\Lambda_{L}\right|^{-\beta}\right)$, up to at most $N\left(J_{j, \Lambda_{L^{\kappa}}}\right)\left|\Lambda_{L^{\prime}}\right|\left|\Lambda_{L}\right|^{-\beta}$ of them, the eigenvalues of $H_{\omega}\left(\Lambda_{L^{\prime}}\right)$ in $J_{j, \Lambda_{L^{\kappa}}}$ are given by those of the operators $\left(H_{\omega}\left(\Lambda_{\ell}(\gamma)\right)_{\gamma}\right)$ up to an error bounded by $|\Lambda|^{-2}$. In particular, up to at most $N\left(J_{j, \Lambda_{L^{\kappa}}}\right)\left|\Lambda_{L^{\prime}}\right|\left|\Lambda_{L}\right|^{-\beta}$ of them, the eigenvalues of $H_{\omega}\left(\Lambda_{L^{\prime}}\right)$ in $J_{j, \Lambda_{L^{\kappa}}}$ with localization center in $\Lambda_{(L-1)^{\nu}}$ and of $H_{\omega}\left(\Lambda_{L^{\kappa}}\right)$ in $J_{j, \Lambda_{L^{\kappa}}}$ with localization center in $\Lambda_{(L-1)^{\nu}}$ are the same up to an error bounded by $C L^{-2 d}$. Moreover, the number of cubes $\left(\Lambda_{\ell}(\gamma)\right)_{\gamma}$ that are not contained in $\Lambda_{(L-1)^{\nu}}$ is bounded by $C L^{\nu d-1}$ which is itself bounded by $C N\left(J_{j, \Lambda_{L^{\kappa}}}\right)\left|\Lambda_{L^{\prime}}\right|\left|\Lambda_{L}\right|^{-\beta}$. Thus, if one pick $p>1$, the Borel-Cantelli Lemma tells us that (1), (3) and (4) of Lemma 3.9 are almost surely fulfilled.

To prove that (2) is also almost surely true, we use the estimates on large deviations given by Theorem 2.2 on the sets $J_{j, \Lambda_{L^{\kappa}}} \backslash K_{j, \Lambda_{L^{\kappa}}}$ that are of size $L^{-d / 2}$. We thus obtain that, with probability at least $1-e^{-L^{d \nu / 4}}$, for $L^{\kappa} \leq L^{\prime} \leq(L+1)^{\nu}$, the number of eigenvalues of of $H_{\omega}\left(\Lambda_{L^{\prime}}\right)$ in $J_{j, \Lambda_{L^{\kappa}}} \backslash K_{j, \Lambda_{L^{\kappa}}}$ is bounded by $N\left(\bar{J}_{j, \Lambda_{L^{\kappa}}}\right)\left|\Lambda_{L^{\prime}}\right| L^{-1}$. Thus, using again the Borel-Cantelli Lemma and (1), we obtain (2).

This completes the proof of Lemma 3.9.

3.6. The proof of Theorem 1.4. It follows the same analysis as the proof of Theorem 1.1; thus, we do not give any details. We distinguish two cases. First if $\left|N\left(I_{\Lambda}\right)\right| \leq$ $|\Lambda|^{-\alpha}$ for $\alpha$ chosen as prescribed in section 3.2 (see also the comments following Theorem 2.1). In this case, we can apply Theorem 2.1 to the interval $I_{\Lambda}$ as it satisfies all the assumptions of Theorem 2.1 if we choose the scales $\ell_{\Lambda} \asymp\left|N\left(I_{\Lambda}\right)\right|^{-\nu}$ for some $\nu$ satisfying (2.8). We then follow the proof of Theorem 1.1 for this single interval to obtain Theorem 1.4. If $\left|N\left(I_{\Lambda}\right)\right| \geq|\Lambda|^{-\alpha}$, we again split the interval into intervals of size $|\Lambda|^{-\alpha}$ to apply Theorem 2.1 to each of those, actually, to most of those. Indeed, up to the renormalization of $N$ so that it has unit mass on $I_{\Lambda}$ we have brought ourselves back to the proof of Theorem 1.1

Remark 3.2. We see that the first condition in (1.8) is needed only when the interval $I_{\Lambda}$ is very small. Actually, one needs it for $\left|I_{\Lambda}\right|$ smaller than $|\Lambda|^{-\nu}$ for some $\nu>0$.

The condition (1.9) is needed to obtain the results corresponding to Lemmas 3.3 and 3.9. In Lemmas 3.3 and 3.9, the error estimate is of size an inverse power of $L$; in the corresponding result in the present setting, it is replaced by $o(1)$ coming from condition (1.9).

3.7. The proof of Theorem 1.5. Theorem 1.5 follows from Theorem 1.1, Lemma 3.4 and the fact that most eigenvalues of $H_{\omega}$ in $J$ with localization center in $\Lambda$ are very well approximated by an eigenvalue of $H_{\omega}(\Lambda)$ in $J$, and vice versa.

Write $J=[a, b]$. Using the techniques of the proof of Lemma 3.9, one proves the following result for the eigenvalues of $H_{\omega}$ is $J$ having localization center in $\Lambda$

Lemma 3.10. Fix $\nu \in(0,1)$. There exists $\beta>0$ such that, $\omega$-almost surely, for $L$ sufficiently large, one has

$$
\left|\frac{N^{f}(J, \Lambda, \omega)}{N(J, \Lambda, \omega)}-1\right| \leq|\Lambda|^{-\beta} ;
$$


(2) to each eigenvalue of $H_{\omega}\left(\Lambda_{L}\right)$ in $J_{L}:=\left[a+L^{-3 d / 2}, b-L^{-3 d / 2}\right]$ with localization center in $\Lambda_{L-L^{\kappa}}$, say, $E$, one can associate an eigenvalue of $H_{\omega}$ in $J$ with localization center in $\Lambda_{L}$, say, $E^{\prime}$, such that $\left|E-E^{\prime}\right| \leq L^{-2 d}$;

(3) to each eigenvalue of $H_{\omega}$ in $J_{L}$ with localization center in $\Lambda_{L-L^{\kappa}}$, say, $E$, one can associate an eigenvalue of $H_{\omega}\left(\Lambda_{L}\right)$ in $J$, say, $E^{\prime}$, that satisfies $\left|E-E^{\prime}\right| \leq L^{-2 d}$.

One then uses this to combine Theorem 1.1 and Lemma 3.4 to obtain Theorem 1.5.

\section{The Proof of Theorems 1.2 And 1.3}

These proofs are simple and rely on general theorems on transformations of point processes (see e.g. [4, Chap. 5.5] and [27, Chap. 3.5]).

4.1. The proof of Theorem 1.2. As in the proof of Theorem 1.1, it suffices to consider the case when $J$ is an interval in the essential support of $\nu$, that is, $N$ is strictly increasing on $J$. In particular, one has $\nu(t)>0$ for almost every $t \in J$.

If $t$ is a random variable distributed according to the law $\nu_{J}(t) d t$, then $\tilde{t}:=N_{J}(t)$ is uniformly distributed on $[0,1]$. Thus, the process $\Xi_{J}(\omega, \tilde{t}, \Lambda)$ under the uniform law in $\tilde{t}$ has the same law as the process $\Xi_{J}\left(\omega, N_{J}(t), \Lambda\right)$ under the law $\nu_{J}(t) d t$.

Rewrite the point measures $\Xi_{J}\left(\omega, N_{J}(t), \Lambda\right)$ and $\tilde{\Xi}_{J}(\omega, t, \Lambda)$ as

$$
\Xi_{J}\left(\omega, N_{J}(t), \Lambda\right)=\sum_{E_{n}(\omega, \Lambda) \in J} \delta_{x_{n}(\omega, t)} \quad \text { and } \quad \tilde{\Xi}_{J}(\omega, t, \Lambda)=\sum_{E_{n}(\omega, \Lambda) \in J} \delta_{\tilde{x}_{n}(\omega, t)}
$$

where

$$
x_{n}(\omega, t):=|N(J)||\Lambda|\left[N_{J}\left(E_{n}(\omega, \Lambda)\right)-N_{J}(t)\right]=|\Lambda|\left[N\left(E_{n}(\omega, \Lambda)\right)-N(t)\right]
$$

and

$$
\tilde{x}_{n}(\omega, t):=\nu(t)|\Lambda|\left[E_{n}(\omega, \Lambda)-t\right] .
$$

Thus, one has

$$
x_{n}(\omega, t)=\varpi_{\Lambda}\left(\tilde{x}_{n}(\omega, t) ; t\right) \quad \text { and } \quad \tilde{x}_{n}(\omega, t)=\chi_{\Lambda}\left(x_{n}(\omega, t) ; t\right)
$$

where

$$
\varpi_{\Lambda}(x ; t)=|\Lambda|\left[N\left(t+\frac{x}{\nu(t)|\Lambda|}\right)-N(t)\right]
$$

and

$$
\chi_{\Lambda}(x ; t)=\nu(t)|\Lambda|\left[N^{-1}\left(N(t)+\frac{x}{|\Lambda|}\right)-t\right]
$$

where $N^{-1}$ is the inverse of the Lipschitz continuous, strictly increasing function $N$. Note that, if $N(J, \Lambda, \omega)$ denotes the number of eigenvalues of $H_{\omega}(\Lambda)$ in $J$, one has

$$
t=\frac{1}{N(J, \Lambda, \omega)} \cdot N^{-1}\left(\sum_{E_{n}(\omega, \Lambda) \in J} N\left(E_{n}(\omega)\right)-\frac{x_{n}}{|\Lambda|}\right) .
$$

Following the notations of [27], let $\mathcal{M}_{p}(\mathbb{R})$ denote the space of point measures on the real line endowed with its standard metric structure. Actually, by Minami's estimate (M), we could restrict ourselves to working with simple point measures.

The point processes $\Xi_{J}\left(\omega, N_{J}(t), \Lambda\right)$ and $\tilde{\Xi}_{J}(\omega, t, \Lambda)$ under the law $\nu_{J}(t) d t$ are the random processes (i.e. the Borelian random variables) obtained as push-forwards of the probability 
measure $\nu_{J}(t) d t$ through the maps $t \in \mathbb{R} \mapsto \Xi_{J}\left(\omega, N_{J}(t), \Lambda\right) \in \mathcal{M}_{p}(\mathbb{R})$ and $t \in \mathbb{R} \mapsto$ $\tilde{\Xi}_{J}(\omega, t, \Lambda) \in \mathcal{M}_{p}(\mathbb{R})$. We denote them respectively by $\Xi_{J}(\omega, \Lambda)$ and $\tilde{\Xi}_{J}(\omega, \Lambda)$.

One can extend the mapping $x \in \mathbb{R} \mapsto \chi_{\Lambda}(x, t) \in \mathbb{R}$ to a map, say, $\chi_{\omega, \Lambda}$ on point measures in $\mathcal{M}_{p}(\mathbb{R})$ on the real line by just mapping the supports pointwise onto one another and computing $t$ using (4.2) i.e.

$$
\chi_{\omega, \Lambda}\left(\sum_{n} a_{n} \delta_{x_{n}}\right)=\sum_{n} a_{n} \delta_{\chi_{\omega, \Lambda}\left(x_{n} ; t\left(\sum_{n} a_{n} \delta_{x_{n}}\right)\right)}
$$

where $t\left(\sum_{n} a_{n} \delta_{x_{n}}\right)$ is defined as

$$
t\left(\sum_{n} a_{n} \delta_{x_{n}}\right)=\frac{1}{N(J, \Lambda, \omega)} \sum_{E_{n}(\omega, \Lambda) \in J} N^{-1}\left(N\left(E_{n}(\omega)\right)-\frac{x_{n}}{|\Lambda|}\right) .
$$

For fixed $\Lambda$ and $\omega$, the map $\chi_{\omega, \Lambda}: \mathcal{M}_{p}(\mathbb{R}) \rightarrow \mathcal{M}_{p}(\mathbb{R})$ is measurable as the map $t \mapsto \chi_{\Lambda}(x, t)$ is. Moreover, by the computations made above (see (4.1) and (4.2)), one has

$$
\chi_{\omega, \Lambda}\left(\Xi_{J}(\omega, \Lambda)\right)=\tilde{\Xi}_{J}(\omega, \Lambda) .
$$

For any $x \in R, t$ almost surely, one has $\chi_{\Lambda}(x ; t) \rightarrow x$ as $|\Lambda| \rightarrow+\infty$. Hence, as $|\Lambda| \rightarrow+\infty$, $\chi_{\omega, \Lambda}$ tends to the identity except on at most a set of measure 0 in $\mathcal{M}_{p}(\mathbb{R})$. On the other hand, Theorem 1.2 tells us that, $\omega$ almost surely, $\Xi_{J}(\omega, \Lambda)$ converges in law to the Poisson process of intensity 1 on the real line. Thus, we can apply [4, Theorem 5.5] to obtain that, $\omega$-almost surely, $\tilde{\Xi}_{J}(\omega, \Lambda)$, that is, $\tilde{\Xi}_{J}(\omega, t, \Lambda)$ under the measure $\nu_{J}(t) d t$, converges in law to the Poisson process of intensity 1 on the real line. This completes the proof of Theorem 1.2.

4.2. The proof of Theorem 1.3. To complete this proof, recalling the notations of Theorem 1.3, we notice that, for $x>0$,

$$
\begin{aligned}
\left\{E_{j} \in J ; \frac{|N(J)|}{|J|}|\Lambda|\left(E_{j+1}(\omega, \Lambda)-E_{j}(\omega, \Lambda)\right) \geq x\right\} & \\
= & \left\{E_{j} \in J ; \nu(t)|\Lambda|\left(E_{j+1}(\omega, \Lambda)-E_{j}(\omega, \Lambda)\right) \geq \nu_{J}(t) \cdot|J| x\right\} .
\end{aligned}
$$

Thus, integration with respect to $\nu_{J}(t) d t$ over $J$, Theorem 1.2 and the same computations as those made to obtain Proposition 4.4 in [24] lead to, $\omega$-almost surely

$$
\begin{aligned}
& D L S(x ; J, \omega, \Lambda)=\int_{J} \frac{\#\left\{E_{j} \in J ; \nu(t)|\Lambda|\left(E_{j+1}(\omega, \Lambda)-E_{j}(\omega, \Lambda)\right) \geq \nu_{J}(t) \cdot|J| x\right\}}{N(J, \omega, \Lambda)} \nu_{J}(t) d t \\
& \rightarrow|\rightarrow| \rightarrow+\infty \\
& \mid{ }_{J} e^{-\nu_{J}(t) \cdot|J| x} \nu_{J}(t) d t .
\end{aligned}
$$

This completes the proof of Theorem 1.3.

\section{REFERENCES}

[1] Michael Aizenman, Alexander Elgart, Serguei Naboko, Jeffrey H. Schenker, and Gunter Stolz. Moment analysis for localization in random Schrödinger operators. Invent. Math., 163(2):343-413, 2006.

[2] Michael Aizenman, Jeffrey H. Schenker, Roland M. Friedrich, and Dirk Hundertmark. Finite-volume fractional-moment criteria for Anderson localization. Comm. Math. Phys., 224(1):219-253, 2001. Dedicated to Joel L. Lebowitz.

[3] Jean V. Bellissard, Peter D. Hislop, and Günter Stolz. Correlation estimates in the Anderson model. J. Stat. Phys., 129(4):649-662, 2007. 
[4] Patrick Billingsley. Convergence of probability measures. Wiley Series in Probability and Statistics: Probability and Statistics. John Wiley \& Sons Inc., New York, second edition, 1999. A WileyInterscience Publication.

[5] Jean Bourgain and Carlos E. Kenig. On localization in the continuous Anderson-Bernoulli model in higher dimension. Invent. Math., 161(2):389-426, 2005.

[6] Jean-Michel Combes, François Germinet, and Abel Klein. Generalized eigenvalue-counting estimates for the Anderson model. J. Stat. Phys., 135(2):201-216, 2009.

[7] Jean-Michel Combes, François Germinet, and Abel Klein. Poisson statistics for eigenvalues of continuum random Schrödinger operators. Anal. PDE, 3(1):49-80, 2010.

[8] François Germinet, Peter D. Hislop, and Abel Klein. Localization at low energies for attractive Poisson random Schrödinger operators. In Probability and mathematical physics, volume 42 of CRM Proc. Lecture Notes, pages 153-165. Amer. Math. Soc., Providence, RI, 2007.

[9] François Germinet, Peter D. Hislop, and Abel Klein. Localization for Schrödinger operators with Poisson random potential. J. Eur. Math. Soc. (JEMS), 9(3):577-607, 2007.

[10] François Germinet and Abel Klein. A characterization of the Anderson metal-insulator transport transition. Duke Math. J., 124(2):309-350, 2004.

[11] Francois Germinet and Abel Klein. New characterizations of the region of complete localization for random Schrödinger operators. J. Stat. Phys., 122(1):73-94, 2006.

[12] François Germinet and Frédéric Klopp. Spectral statistics for random Schrödinger operators in the localized regime. ArXiv http://arxiv.org/abs/1011.1832, 2010.

[13] F. Ghribi, P. D. Hislop, and F. Klopp. Localization for Schrödinger operators with random vector potentials. In Adventures in mathematical physics, volume 447 of Contemp. Math., pages 123-138. Amer. Math. Soc., Providence, RI, 2007.

[14] Fatma Ghribi and Frédéric Klopp. Localization for the random displacement model at weak disorder. Ann. Henri Poincaré, 11(1-2):127-149, 2010.

[15] Gian Michele Graf and Alessio Vaghi. A remark on the estimate of a determinant by Minami. Lett. Math. Phys., 79(1):17-22, 2007.

[16] Peter D. Hislop. Lectures on random Schrödinger operators. In Fourth Summer School in Analysis and Mathematical Physics, volume 476 of Contemp. Math., pages 41-131. Amer. Math. Soc., Providence, RI, 2008.

[17] Peter D. Hislop and Frédéric Klopp. The integrated density of states for some random operators with nonsign definite potentials. J. Funct. Anal., 195(1):12-47, 2002.

[18] Werner Kirsch. An invitation to random Schrödinger operators. In Random Schrödinger operators, volume 25 of Panor. Synthèses, pages 1-119. Soc. Math. France, Paris, 2008. With an appendix by Frédéric Klopp.

[19] Werner Kirsch and Bernd Metzger. The integrated density of states for random Schrödinger operators. In Spectral theory and mathematical physics: a Festschrift in honor of Barry Simon's 60th birthday, volume 76 of Proc. Sympos. Pure Math., pages 649-696. Amer. Math. Soc., Providence, RI, 2007.

[20] Frédéric Klopp. Localization for some continuous random Schrödinger operators. Comm. Math. Phys., 167(3):553-569, 1995.

[21] Frédéric Klopp, Michael Loss, Shu Nakamura, and Günter Stolz. Localization for the random displacement model, 2010. ArXiv http://fr.arxiv.org/abs/arXiv:1007.2483.

[22] Nariyuki Minami. The energy level statistics for the Anderson tight binding model - statement of a conjecture. Online document: www.math.h.kyoto-u.ac.jp/ ueki/SR06/minami.pdf.

[23] Nariyuki Minami. Local fluctuation of the spectrum of a multidimensional Anderson tight binding model. Comm. Math. Phys., 177(3):709-725, 1996.

[24] Nariyuki Minami. Theory of point processes and some basic notions in energy level statistics. In Probability and mathematical physics, volume 42 of CRM Proc. Lecture Notes, pages 353-398. Amer. Math. Soc., Providence, RI, 2007.

[25] Nariyuki Minami. Energy level statistics: a formulation and some examples. In N. Minami, editor, Spectra of random operators and related topics, 2011. To appear.

[26] Leonid Pastur and Alexander Figotin. Spectra of random and almost-periodic operators, volume 297 of Grundlehren der Mathematischen Wissenschaften [Fundamental Principles of Mathematical Sciences]. Springer-Verlag, Berlin, 1992.

[27] Sidney I. Resnick. Extreme values, regular variation and point processes. Springer Series in Operations Research and Financial Engineering. Springer, New York, 2008. Reprint of the 1987 original. 
[28] Peter Stollmann. Caught by disorder, volume 20 of Progress in Mathematical Physics. Birkhäuser Boston Inc., Boston, MA, 2001. Bound states in random media.

[29] Ivan Veselić. Existence and regularity properties of the integrated density of states of random Schrödinger operators, volume 1917 of Lecture Notes in Mathematics. Springer-Verlag, Berlin, 2008.

(Frédéric Klopp) LAGA, U.M.R. 7539 C.N.R.S, Institut Galilée, Université Paris-Nord, 99 Avenue J.-B. Clément, F-93430 Villetaneuse, France

E-mail address: klopp@math.univ-paris13.fr 